ENCYCLOPÉDIE Encyclopédie berbère

BERBERE

5 | 1988

5 | Anacutas - Anti-Atlas

\title{
Anthropologie (Partie III)
}

M.-C. Chamla et D. Ferembach

\section{OpenEdition}

Journals

Édition électronique

URL : http://journals.openedition.org/encyclopedieberbere/2897

DOI : 10.4000/encyclopedieberbere.2897

ISSN : 2262-7197

\section{Éditeur}

Peeters Publishers

\section{Édition imprimée}

Date de publication : 1 avril 1988

Pagination : 713-775

ISBN : 2-85744-379-6

ISSN : 1015-7344

\section{Référence électronique}

M.-C. Chamla et D. Ferembach, « Anthropologie (Partie III) », Encyclopédie berbère [En ligne], 5 | 1988,

document A234, mis en ligne le 01 décembre 2012, consulté le 12 octobre 2020. URL : http://

journals.openedition.org/encyclopedieberbere/2897; DOI : https://doi.org/10.4000/

encyclopedieberbere. 2897

Ce document a été généré automatiquement le 12 octobre 2020

(c) Tous droits réservés 


\title{
Anthropologie (Partie III)
}

\author{
M.-C. Chamla et D. Ferembach
}

\section{Anthropologie physique des Marocains (D. Ferembach)}

\section{1. - Les Rifains}

1 Leur étude a été réalisée par C.S. Coon (1931, 1939). Les Rifains sont essentiellement des agriculteurs sédentaires. Seuls les Métalsa et les Beni Bu Yahyi, au sud-est du Rif, mènent une vie de nomade dans le désert de Garet. Les tribus étudiées par C.S. Coon sont les suivantes: Kebdana, Galiya, Beni Said, Beni Bu Yahyi, Metalsa, Beni Ulinshk, Tafersit, Beni Tuzin, Temsaman, Geznaya, Beni Urriaghel, Beni Amart, Targuist, Bokoya, Beni Itteft, Beni Bu Frah, Mestabsa et Mtiwa. En moyenne, les Rifains ont une stature modérée, une tête allongée et élevée et un nez leptorhinien.

2 Leur stature moyenne est de $168,6 \mathrm{~cm} \pm 0,2$. Parmi les diverses tribus examinées, cette moyenne varie entre $166,2 \mathrm{~cm}$ (chez les Targuist) et $171,5 \mathrm{~cm}$ (chez les Tuzin). La grande envergure atteint une moyenne de $175,4 \mathrm{~cm} \pm 0,2$ et l'indice rapportant cette mesure à la stature $104,1 \pm 0,1$. La taille assis s'élève à $85,4 \pm 0,1$ et l'indice correspondant à $50,9 \pm 0,1$.

3 En général, « les épaules sont modérément larges et les hanches étroites » (diamètre moyen biacromial $=37,3 \mathrm{~cm} \pm 0,1$, diamètre moyen bi-iliac $=29,6 \mathrm{~cm} \pm 0,1)$. On rencontre aussi un type d'aspect plus massif, avec des épaules et des hanches larges et un tronc allongé, mais sa fréquence est bien moindre.

4 Comme pour la stature, les têtes possédant les plus grandes dimensions se trouvent à l'est du Rif. C'est ainsi que les Kebdana ont une longueur moyenne de la tête de 197,9 $\mathrm{mm} \pm 0,1$ alors que chez les Bokoya et les Urriaghel elle n'atteint que 191,4 et 191, $7 \mathrm{~mm}$. Pour l'ensemble du Rif, cette mesure est de 194,5 $\mathrm{mm} \pm 0,2$, la largeur moyenne de la tête étant de $145,8 \mathrm{~mm} \pm 0,2$ (les moyennes entre les tribus s'étagent entre 143 et $148 \mathrm{~mm}$ : $143,2 \mathrm{~mm} \pm 0,6 \mathrm{chez}$ les Bokoya, 148,3 $\mathrm{mm} \pm 0,5 \mathrm{chez}$ les Mazuza). 
5 L'indice céphalique horizontal varie peu entre les tribus et reste toujours dans les limites de la dolichocéphalie (73,5 $\pm 0,6 \mathrm{chez}$ les Kebdana, 75,8 $\pm 0,3 \mathrm{chez}$ les Temsamam).

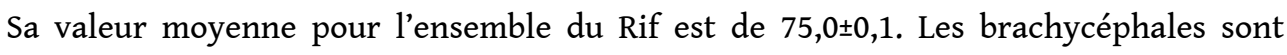
rares dans cette population.

6 La tête se montre élevée $(M=129,2 \mathrm{~mm} \pm 0,2$, écart entre les moyennes $127,2-131,5$ $\mathrm{mm})$. Tous les indices de hauteur-longueur se classent dans la catégorie hypsicéphale $(M=66,8 \pm 0,1)$. L'indice de hauteur-largeur indique lui aussi une tête haute, acrocéphale $(\mathrm{M}=88,7 \pm 0,1)$.

Le diamètre frontal minimum atteint la moyenne de 106,1 $\mathrm{mm} \pm 0,1(\mathrm{~W}=103,8-108,5$ $\mathrm{mm})$. L'indice fronto-pariétal confirme la largeur du front $(M=72,9 \pm 0,1)$.

7 La face des Rifains présente des dimensions moyennes. Au rapport entre la hauteur totale de la face $(M=124,1 \mathrm{~mm} \pm 0,2 ; \mathrm{W}=121,2-131,2 \mathrm{~mm})$ et le diamètre bizygomatique $(\mathrm{M}=136,0 \mathrm{~mm} \pm 0,1 ; \mathrm{W}=132,7-140,0 \mathrm{~mm})$ correspond un indice facial leptoprosope ( $M=91,4 \pm 0,2$; écart entre les moyennes des différentes tribus, $W=88,6$ $95,9)$. Les faces les plus allongées se rencontrent parmi les tribus de l'est, les plus courtes "parmi les tribus centrales de plus pures traditions rifaines", dont l'indice moyen se rapproche de la mésoprosopie.

8 La largeur bigoniale est plus faible que la largeur bizygomatique $(\mathrm{M}=106,1 \mathrm{~mm} \pm 0,2$; $\mathrm{W}=103,6-109,5 \mathrm{~mm})$. Le nez, leptorhinien dans toutes les séries $(\mathrm{M}=63,6 \pm 0,2 ; \mathrm{W}=$ $61,2-64,9 \mathrm{~mm})$ possède une hauteur moyenne de 54,7 $\pm 0,1 \mathrm{~mm}(\mathrm{~W}=52,7-56,9 \mathrm{~mm})$ et une largeur moyenne de 34,6 $\mathrm{mm} \pm 0,1(\mathrm{~W}=33,9-35,6 \mathrm{~mm})$. L'indice diminue de l'est vers l'ouest en même temps que les dimensions du nez augmentent.

9 La couleur de leur peau, relevée sur la poitrine et sur la face interne de l'avant-bras, s'est montrée claire dans $65 \%$ des cas, ce pourcentage variant entre $85,9 \%$ chez les Ouriagels et $44,8 \%$ chez les Nomades. Une pigmentation foncée n'a été observée que chez $4,3 \%$. Le nombre d'individus porteurs d'éphélides (taches de rousseur) s'élève à $22,7 \%$. Les cheveux qui sont ondulés ou bouclés se montrent un peu plus foncés que la peau puisque ils ont été trouvés noirs chez $43,7 \%$ des sujets examinés et bruns foncés chez $25 \%$; elle tend vers le roux chez $17 \%$ d'entre eux. Les yeux bruns foncés dominent ( $43 \%)$; les autres sont soit gris brun, soit brun vert, soit brun bleu, soit gris $(0,2 \%)$ ou bleu $(1,9 \%)$.

10 De ces observations, C.S. Coon conclut à la fréquence du blondisme dans le Rif, la moitié des habitants en présentant des traces dans la coloration soit de leurs cheveux, soit de leur barbe, soit de leurs yeux. Selon lui, 4 types principaux peuvent se reconnaître parmi les Rifains.

11 «L'un est de type à face allongée, à nez busqué, à pigmentation brune foncée ou mélangée, plus commun dans le nord-est, en particulier parmi les Nomades; cette physionomie est considérée par les Rifains comme une importation de Zénata ou d'inspiration Arabe. Un autre est un Méditerranéen classique, avec un front légèrement fuyant, un profil nasal rectiligne, à extrémité légèrement relevée, avec des ailes du nez moyennes et un contour facial ovale; ce type est généralement brun foncé; on le trouve partout, mais particulièrement parmi les tribus du centre et de l'ouest. Un troisième est un Nordique dans le sens morphologique le plus strict, avec généralement des cheveux bruns et des yeux mélangés, et un « Nordique Rifain pourrait être pris pour un Irlandais ou un Anglais, moins facilement pour un Scandinave ». 
12 « Un quatrième est un type au corps large, à la tête large tendant vers la mésocéphalie, avec un aplatissement lambdoïdien marqué, particulièrement visible quand la tête est rasée; la face est large, les orbites basses, comme on peut facilement le discerner à partir de l'ouverture palpébrale étroite et la présence de pli palpébral médian ou externe; le nez est court, droit ou parfois relevé, la bouche large, le menton proéminent et la mâchoire massive. La pigmentation est en général mélangée, avec des cheveux bruns ou roux et des yeux clairs-mélangés. Ce type, sans contexte, est évidemment un survivant moderne quelque peu atténué des vieux hommes d'Afalou ressemblant aux Cro-Magnons. On le trouve principalement parmi les familles rifaines les plus anciennes et parmi les fonctionnaires tribaux. Ils ne sont pas rares dans le Rif...»

13 «Une variante plus petite de celui-ci est considérée par les Rifains eux-mêmes comme typique des Beni Urriaghel de la montagne; de petite stature, de constitution large, avec des mains courtes et larges, une peau présentant des taches de rousseur, un barbe rousse, des yeux bleus, une face courte et large avec une mâchoire carrée et un nez camus. C'est la prédominance de ce dernier type, concentré dans le groupe montagneux entre les Beni Urriaghel et les Geznaya.

14 Les pourcentages suivants ont été obtenus pour les groupes sanguins A, B, O. On ne dispose pas de données sur les autres systèmes de groupes érythrocytaires :

\begin{tabular}{|c|c|c|c|c|c|}
\hline $\mathrm{N}$ & $\mathrm{O}$ & $\mathrm{A}$ & $\mathrm{B}$ & $\mathrm{AB}$ & Auteurs \\
\hline 195 & 50,26 & 18,36 & 28,72 & 3,08 & C.S. Coon, 1931 \\
165 & 59,39 & 24,85 & 14,55 & 1,21 & J. Gaud, 1942 \\
\hline
\end{tabular}

\section{2. - Tribus au sud et à l'est du Rif}

15 (Bassin du Sebou, Trouée de Taza, Mts des Beni Snassen).

Ces tribus occupent les plaines au sud du Rif, jusqu'à une ligne passant sensiblement au Nord des chaînes de l'Atlas, par Rabat et Fez.

N. Kossovitch (1953) a étudié dans cette région les tribus suivantes: Beni Ahsen, Beni Malek, Sefiane du Gharb, Cherarda, Beni Mestara, Beni Zeroual, Beni Urriaghel et Beni Mesquilda, Mtiou, Mesraoua, Meziat, Rioua et Senhadja de Mosbah, Senhadja de Gheddon, Confédération Hayaina (Oulad Riab, Oulad Aliane), Brane, Tsoul, Riata, Gueznaia, Metalsa, Haouara, Beni Snassen.

17 Suivant les groupes, la moyenne de la stature varie entre 72,2 et 74,7 , celle de l'indice de hauteur-longueur entre 65,6 et 69,6, celle de l'indice de hauteur-largeur entre 89,4 et 95,7 ; la moyenne de l'indice facial se situe entre 91,4 et 94,4 , celle de l'indice nasal entre 63,7 et 68,8 .

Les hommes appartenant à ces tribus sont donc, en moyenne, de stature moyenne à sur-moyenne, dolichocéphale, avec une tête haute aussi bien par rapport à sa longueur que par rapport à sa largeur, une face leptoproscope et même hyperleptoproscope, un nez leptorhinien. Le pourcentage d'yeux clairs varie entre 10,3 et $20,7 \%$; celui des cheveux clairs entre 5,9 et $24,0 \%$. Le front est décrit comme haut ou moyennement élevé, vertical ou légèrement fuyant, les oreilles assez grandes et écartées de la tête (ce caractère étant dû au port d'une coiffure); le nez, le plus souvent droit et à dos rectiligne, peut prendre une forme légèrement busquée ou « arménoïde »; les lèvres se 
montrent épaisses, la bouche grande ou moyenne; le menton saille de façon variable, mais souvent fortement.

Cet aperçu général montre seulement qu'un élément méditerranéen y prédomine nettement. Mais est-il seul représenté ?

Une réponse à cette question nous sera suggérée, entre autres, par l'examen de la répartition des individus dans les classes des différents indices considérés.

Nous prendrons comme exemple quelques tribus.

Les Senhadja de Geddou sont probablement d'origine saharienne ; il descendraient de ces nomades qui ont donné naissance à la dynastie almoravide. La moyenne de leur stature est la plus faible de toutes les tribus étudiées par N. Kossovitch $(\mathrm{M}=164 \mathrm{~cm}+$ 7,$2 ; \mathrm{W}=150-180 \mathrm{~cm}$ ) et ils possèdent le plus petit diamètre antéro-postérieur de la tête $(\mathrm{M}=18,4 \mathrm{~cm})$. La quasi-totalité des têtes est dolicéphale $(85 \%)$, un petit nombre mésocéphale (14\%); les brachycéphales ne représentent que $1 \%$ de la population. Ces hommes se distinguent aussi par une grande fréquence de leptoprosopie et d'hyperleptoprosopie ( $88 \%$ des cas). Le nez se montre étroit chez $84 \%$ des individus, les platyrhiniens laissant supposer un apport négroïde ne représentant que $1 \%$ de la population. Les yeux et les cheveux clairs sont relativement fréquents chez eux (respectivement $16 \%$ et $19 \%$ ). On peut donc admettre que les Méditerranéens dominent dans cette tribu qui comportait aussi quelques Nordiques.

Les Senhadja Sghir semblent présenter davantage de caractères négrö̈des.

A côté des Senhadja, nous citerons les Beni Mestara (mais ils appartiennent plutôt aux Rifains) et les Beni Zerroual qui se signalent par une plus grande proportion de faces chamaeprosopes (11,8\%). Ils appartiennent au clan des Ghomara, probablement d'ancienne souche berbère.

Leur tête est particulièrement allongée : on dénombre $88,2 \%$ d'hyperdolichocéphales et de dolicéphales. Leur nez est en majorité leptorhinien (69\%); on ne compte que 1,4\% de platyrhiniens. Leur stature se montre moyenne à grande

Il semble que chez eux existe une certaine proportion de descendants d'Hommes de Mechta-Afalou, présentent un pourcentage faible, mais néanmoins plus élevé que dans les autres tribus, de brachy et sous-brachycrânes. Les nez mésorhiniens s'y montrent aussi plus fréquents. Faut-il y avoir l'indice d'Alpins?

27 Nous avons déjà noté la proportion relativement élevée d'yeux et de cheveux clairs chez les Senhadja de Geddou. Des chiffres comparables et même plus forts se rencontrent chez les Fassi, chez les Beni Urriaghel, chez les Beni Mesguida, chez les Metalsa : les pourcentages arrivent à dépasser le cinquième de la population, laissant supposer un apport nordique s'ajoutant à l'élément blond qui se rencontre normalement chez les Méditerranéens. mêmes composantes raciales que dans le Rif. L'élément méditerranéen y domine ; on y constate aussi la présence, avec une fréquence variable, suivant les tribus, d'éléments alpins (en faible nombre), nordiques ou de type de Mechta Afalou. Un apport de sang noir se décèle en très faible proportion et sous forme de métissage.

On doit à M. Gessain (1957) l'étude des crêtes papillaires digitales de 194 hommes de la tribu des Beni Mathar habitant non loin de la frontière algérienne dans la région de Buerguent. Ces hommes sont « de grands dolichocéphales bruns et appartiennent à une 
variété de grande taille de la race méditerranéenne » (M. Gessain, 1957). En ce qui concerne les dermatoglyphes, on constate que les arcs sont relativement peu nombreux $(3,1 \%)$ et que les boucles $(57,9 \%)$ l'emportent sur les tourbillons $(38,8 \%)$.

Plusieurs auteurs se sont intéressés aux groupes sanguins d'un certain nombre de tribus. Il n'est pas possible de reproduire ici tous les résultats publiés. Nous donnons cidessous les pourcentages des groupes A, B, O, obtenus en tenant compte des données de N. Kossovitch et ceux correspondant aux valeurs relevées par A.E. Mourant et alii (1958) chez divers auteurs :

\begin{tabular}{|c|c|c|c|c|c|c|c|c|c|}
\hline & \multicolumn{2}{|c|}{$\mathrm{O}$} & \multicolumn{2}{|c|}{$\mathrm{A}$} & \multicolumn{2}{|c|}{ B } & \multicolumn{2}{|c|}{$\mathrm{AB}$} & \\
\hline \multirow[b]{2}{*}{$\begin{array}{l}\text { Kossovitch } \\
\text { Mourant }\end{array}$} & $\mathrm{N}$ & $\%$ & & $\%$ & $\mathrm{~N}$ & $\%$ & $\mathrm{~N}$ & $\%$ & N Total \\
\hline & $\begin{array}{r}903 \\
2202 \\
\end{array}$ & $\begin{array}{l}43,41 \\
45,0\end{array}$ & $\begin{array}{r}744 \\
1563 \\
\end{array}$ & $\begin{array}{l}35,77 \\
31,9\end{array}$ & $\begin{array}{l}347 \\
880\end{array}$ & $\begin{array}{l}16,68 \\
18,0\end{array}$ & $\begin{array}{r}86 \\
253\end{array}$ & $\begin{array}{l}4,14 \\
5,2\end{array}$ & $\begin{array}{l}2080 \\
4898\end{array}$ \\
\hline
\end{tabular}

31 On constate une relative concordance entre eux.

32 Pour les système erythrocytaires $\mathrm{MN}$ et $\mathrm{Rh}$, les pourcentages calculées à partir des données relevées par A. Messerlin (in A.E. Mourant 1954) sur les 5 groupes qu'il a examinés sont les suivantes :

\begin{tabular}{|c|cccc|cc|}
\hline & \multicolumn{3}{|c|}{ Phénotypes \% } & \multicolumn{2}{c|}{ Gènes $\%$} \\
\hline N Total & & MM & MN & NN & M & N \\
& \% observés & 25,80 & 53,14 & 21,05 & 52,44 & 47,56 \\
& \% théorique & 27,58 & 49,59 & 22,83 & & \\
\hline N Total & & $\mathrm{Rh}+$ & $\mathrm{Rh}-$ & & $\mathrm{D}$ & $\mathrm{d}$ \\
1872 & & 86,75 & 13,25 & & 63,78 & 36,22 \\
\hline
\end{tabular}

\section{3. - Les arabophones de la côte atlantique}

Nous regroupons sous cette appellation les tribus qui occupent la région côtière entre Rabat et Mogador. Celles examinées par N. Kossovitch (1953) sont les suivantes : Zaër, Chaouia, Doukkala, Ahmar, Rehamna, Seraghna, Abda, Guich, Chiadma, Beni Zemmour, Beni Moussa, Beni Amir, Ourdigha, Smala, Oulad Bou Sebaa, Mejjat et Frouga. Les groupes sanguins A, B, O, seuls groupes érythrocytaires sur lesquels on ait des données, sont représentés avec les fréquences ci-dessous :

\begin{tabular}{|c|c|rc|cc|cc|cc|}
\hline \multicolumn{2}{|c|}{} & \multicolumn{2}{|c|}{ O } & \multicolumn{2}{c|}{ A } & \multicolumn{3}{c|}{ B } & \multicolumn{3}{c|}{ AB } \\
\hline N Total & Auteur & \multicolumn{1}{|c|}{ N } & $\%$ & N & $\%$ & N & $\%$ & N & $\%$ \\
\hline 833 & Kossovitch & 355 & 42,62 & 290 & 34,81 & 155 & 18,61 & 33 & 3,96 \\
2552 & Mourant & 1262 & 49,45 & 791 & 31,00 & 413 & 16,18 & 86 & 3,37 \\
\hline
\end{tabular}

Les dimensions moyennes de ces hommes, et le pourcentage d'individus aux yeux et aux cheveux clairs, sont comparables à celles des Bérabers ou des tribus du Nord. Suivant les groupes considérés, la stature moyenne varie entre 166 et $170 \mathrm{~cm}$, l'indice crânien horizontal entre 73,2 et 75,9, l'indice facial entre 90,2 et 93,9 et l'indice nasal entre 62,6 et 68,7 . Proche de la mésorhinie, cette dernière valeur se rencontre chez les tribus installées sur le versant sud du Haut-Atlas: Oulad Bou Sebaa, Mejjat, Frouga. C'est chez elles aussi que l'on trouve le pourcentage de nez platyrhiniens le plus élevé $(4,5 \%)$, signalant un plus grand apport de sang noir. Celui-ci reste relativement large, à 
moins que ce caractère ne soit dû à une plus grande fréquence de descendants d'Ibéromaurusiens?

\section{4. - Les Bérabers}

Ils vivent sur les Hauts Plateaux, sur les pentes du Moyen Atlas et dans les bassins de la Moulouya. Ils sont soit sédentaires, soit semi-nomades, pratiquant la transhumance. Les tribus étudiées par N. Kossovitch (1953) sont les suivantes: Confédération Zemmour, Zaïan, Aït Roboa, Beni Mtir, Beni Mguild, Aït Ayach, Aït Yzdeg, Ait Ouafellah, Aït Youssi, Ait Youssi-Ketchala-Pachalik, Ait Seghrouchen (Aït Seyrušen), Aït Seghrouchen d'Immouzer, Aï Seghrouchen de Sidi Ali ou du Djebel Tichoukt, Aït Seghrouchen de Harira, Marmoucha, Beni Ouaraïn, les Bahloula.

Leur stature varie entre 165 et $169 \mathrm{~cm}$ suivant les tribus, et l'indice céphalique horizontal entre 71,5 et 76,1, les indices de hauteur de leur tête indiquent tous des calottes élevées. L'indice facial (dont les moyennes oscillent entre 91,6 et 96,3) se situent toujours, en moyenne, dans les marges de la leptorhinie. On constate un pourcentage de cheveux et d'yeux clairs un peu plus élevé que chez les tribus du Nord.

Les groupes sanguins $A, B, A B, O$ se rencontrent avec les pourcentages donnés cidessous. On ne possède pas de données sur les autres systèmes des groupes érythrocytaires.

\begin{tabular}{|l|cc|cc|rc|cc|cc|}
\hline & \multicolumn{2}{|c|}{$\mathrm{O}$} & \multicolumn{2}{c|}{ A } & \multicolumn{2}{c|}{ B } & \multicolumn{2}{c|}{ AB } & \multicolumn{2}{c|}{ Total } \\
\hline & $\mathrm{N}$ & $\%$ & $\mathrm{~N}$ & $\%$ & $\mathrm{~N}$ & $\%$ & $\mathrm{~N}$ & $\%$ & $\mathrm{~N}$ & $\%$ \\
Kossovitch & 364 & 40,81 & 347 & 38,90 & 141 & 15,81 & 40 & 4,48 & 892 & 100 \\
Mourant & 125 & 32,81 & 147 & 38,58 & 89 & 23,36 & 20 & 5,25 & 381 & 100
\end{tabular}

L'élément méditerranéen dans ses deux variétés, robuste et gracile, domine toujours dans ce groupe. Mais la proportion de descendants des Ibéromaurusiens du type de Mechta-Afalou et de Brachycrânes alpins issus soit de ces derniers soit des Méditerranéens, semble plus importante que chez les tribus arabophones et berbérophones du Nord, vivant dans les plaines au sud du Rif.

\section{5. - Les Chleuhs}

Le nom de Chleuhs a été donné aux Berbères parlant le tašelyit et vivant dans les montagnes du Haut Atlas de Marrakech et de l'Anti-Atlas occidental, à partir de la côte atlantique, au Sud de la ville de Mogador. Vers le Sud, leur limite est imprécise et se perd dans le Sahara. Ces hommes mènent une vie soit sédentaire soit nomade, pratiquant alors la transhumance dans les montagnes.

41 Les tribus étudiées par N. Kossovitch (1953) sont: Haha, Confédération Mesfioua, IdaOu-Tanan, Ida-Ou-Ziki, Ida-Ou-Zal, Ida-Ou-Mahmoud, Erguita, Mentaga, Aït-Igges, IdaOu-Zedagh, Goundafa, Glaoua, Zemran, Ftouaka, Oultana, Entifa.

Les Chleuhs ne forment pas plus que les autres ethnies marocaines, une population anthropologiquement homogène.

La région du Sous doit être considérée à part. Elle forme une enclave qui « comprend outre la vallée du Sous, l'extrémité occidentale du Grand Atlas et une partie du versant sud de ce massif, avec le massif du Siroua et le massif montagneux de l'Anti-Atlas »... 
«Cette population comprend des Berbères, des Arabes et des Soudanais fortement mélangés les uns aux autres. Il y a aussi un assez grand nombre de juifs ». (N. Kossovitch, 1953). Les tribus leur correspondant sont: Ksima, Mes-guina, Haouara, Oulad, Yahia, Menabha, Guettioua, Chtouka.

Chez les Chleuhs, la stature, suivant les tribus, varie en moyenne entre 165 et $169 \mathrm{~cm}$, les mesures individuelles s'étagent entre 152 et $187 \mathrm{~cm}$. L'indice céphalique est essentiellement dolicéphale $(M=73,8$ à 75,6 mesures individuelles, $W=59,8$ à 86,0$)$ et l'indice facial lepto ou hyperléptoprosope $(\mathrm{M}=89,5$ à 95,2 mesures individuelles $\mathrm{W}=$ 80,1 - 102,2). L'indice nasal se montre, en moyenne, soit lepto, soit mésorhinien (70,5 chez les Mesguina du Sous, 72,2 chez les habitants de Marrakech).

Lorsque l'on tient compte des pourcentages de répartition des indices on constate, par rapport aux Bérabers, une augmentation des brachycrânes, des faces chamaeprosopes, rectangulaires, basses et larges, des nez platyrhiniens. La proportion des yeux et des cheveux clairs est au contraire en régression (valeurs extrêmes des pourcentages d'yeux clairs : 7,7 \% et 18,3\%; pour les cheveux clairs, $3,4 \%$ et $20,0 \%$ ).

Une plus grande fréquence de métissage avec les Noirs, due à la proximité avec l'Afrique noire, peut rendre compte de ces pourcentages. Il est certain aussi que la frontière commune et imprécise avec le Sahara a facilité les croisements avec les Nomades.

Quoi qu'il en soit, les mêmes composantes raciales que dans les autres régions du Maroc se retrouvent: Méditerranéens, quelques Nordiques, des Alpins issus soit de Méditerranéens soit d'Ibéromaurusiens.

Nous donnons ci-dessous des moyennes des pourcentages établis par N. Kossovitch (1953) pour les groupes sanguins A,B,0, des Chleuhs et celles calculées à partir des valeurs publiées par A.E. Mourant (1958) :

\begin{tabular}{|l|cc|cc|cc|cc|c|}
\hline & \multicolumn{2}{|c|}{$\mathrm{O}$} & \multicolumn{2}{|c|}{$\mathrm{A}$} & \multicolumn{2}{|c|}{$\mathrm{B}$} & \multicolumn{2}{|c|}{$\mathrm{AB}$} & \\
\hline & $\mathrm{N}$ & $\%$ & $\mathrm{~N}$ & $\%$ & $\mathrm{~N}$ & $\%$ & $\mathrm{~N}$ & $\%$ & $\mathrm{~N}$ total \\
Kossovitch & 477 & 43,9 & 369 & 34,0 & 185 & 17,1 & 54 & 5,0 & 1085 \\
Mourant & 1019 & 61,09 & 381 & 22,84 & 233 & 13,97 & 35 & 2,1 & 1668 \\
\hline
\end{tabular}

Pour les tribus du Sous, N. Kossovitch a obtenu des résultats suivantes :

\begin{tabular}{|c|c|c|c|c|c|c|c|c|}
\hline \multicolumn{2}{|c|}{0} & \multicolumn{2}{|c|}{ A } & \multicolumn{2}{|c|}{ B } & \multicolumn{2}{|c|}{$\mathrm{AB}$} & \\
\hline $\mathrm{N}$ & $\%$ & $\mathrm{~N}$ & $\%$ & $\mathrm{~N}$ & $\%$ & & $\%$ & $\mathrm{~N}$ total \\
\hline 141 & 42,7 & 114 & 34,6 & 60 & 18,2 & 15 & 4,5 & 330 \\
\hline
\end{tabular}

On constate sur tous les tableaux une prédominance du groupe 0 , les autres groupes décroissant dans l'ordre A, B, AB. Plusieurs tribus du Haut Atlas se caractérisent par une forte fréquence du groupe 0 ; celle-ci atteint $81,8 \%$ chez les Ait Moghrad et 78,2 \% chez les Aït Haddidou. La dérive génétique pourrait rendre compte de ces résultats, ces tribus vivant assez isolées. Elles se distinguent d'ailleurs aussi par les fréquences particulières présentées par les groupes $\mathrm{Rh}-\left(29,9 \%\right.$ soit $55 \%$ de gènes $\mathrm{d}$ pour la $\mathrm{l}^{\mathrm{re}}$, $28,7 \%$ soit $54 \%$ de gène $d$ pour la $2^{2}$ ) et $M N$ ( $18 \%$ de $M$ pour la première).

Les moyennes obtenues pour ces deux systèmes érythrocytaires à partir des examens sérologiques faits par A. Messerlin (in. A.E. Mourant, 1954) sont indiquées ci-dessous. Les tribus examinées par cet auteur sont les Aït Haddidou, les Aït Moghrab, les Aït 
Izdek, qualifiés par lui de Berbères, les Chorfa d'Assoul et les Chorfa de Sidi Boukil (que les groupes Rh pour ces derniers), qualifiés d'Arabes.

\begin{tabular}{|c|ccc|cc|}
\hline & \multicolumn{3}{|c|}{ Phénotypes \% } & \multicolumn{2}{c|}{ Gènes $\%$} \\
\hline $\mathrm{N}$ total & $\mathrm{MM}$ & $\mathrm{MN}$ & $\mathrm{NN}$ & $\mathrm{M}$ & $\mathrm{N}$ \\
627 & Obs.(1) 10,85 & 42,10 & 47,05 & 31,74 & 68,26 \\
& The (1) 11,32 & 41,15 & 47,53 & & \\
\hline $\mathrm{N}$ total & $\mathrm{Rh}+$ & $\mathrm{Rh}-$ & & $\mathrm{D}$ & $\mathrm{d}$ \\
1012 & $76,48 \%$ & $23,52 \%$ & & $52,17 \%$ & $47,92 \%$ \\
\hline
\end{tabular}

(1) Obs. : pourcentage observés

The : pourcentage théorique.

\section{Anthropologie physique des Algériens (M.-C. Chamla)}

\section{Caractères métriques et descriptifs}

L'étude des caractères morphologiques (caractères métriques et descriptifs, Chamla, 1974) d'Algériens originaires de régions de plaines et de montagnes de la zone septentrionale de l'Algérie montre qu'il existe un brassage particulièrement intense dans la population, si bien que les distances anthropologiques globales entre les séries régionales étudiées apparaissent faibles. Néanmoins, l'analyse typologique régionale révèle l'existence d'éléments variés au sein de la population, et dont les caractéristiques indiquent que certaines modifications doivent être apportées aux conceptions classiques sur le peuplement actuel de l'Algérie.

Trois types fondamentaux sont présents dans toutes les séries régionales étudiées dans des proportions variant généralement assez peu, fait qui expliquerait cette faiblesse des coefficients de distance entre les régions. (Les coefficients de distance ont été calculés selon la formule g de Hiernaux - tableau I). L'élément méditerranéen représente l'élément majeur (environ les $3 / 4$ de la population) où l'on distingue trois variantes :

a) un type ibéro-insulaire et un type sud-oriental dont le signalement est suffisamment proche pour qu'ils n'aient pu être séparés par le procédé de l'analyse typologique, et dont les caractéristiques sont essentiellement une stature petite ou moyenne, la dolichocéphalie, la méso-à l'hyperleptoprosopie de la face ;

b) un type atlanto-méditerranéen aussi représenté que le précédent, plus robuste et de stature plus élevée, mésocéphale, à la face longue à très longue ;

c) un type dit "saharien ", moins fréquent (15 à $20 \%$ des sujets), de stature élevée, dolichocéphale, à face longue à très longue.

Un second élément qualifié d'alpin par les auteurs en raison de sa brachycéphalie, de sa face courte et de sa stature peu élevée, existe dans la population dans une proportion d'environ $1 / 10^{e}$, mais il ne semble pas qu'on puisse le confondre pour autant avec le type alpin européen, ne serait-ce qu'en raison de l'atténuation très marquée de sa brachycéphalie, du développement latéral de ses mâchoires et de la forme fréquemment quadrangulaire de sa face, tous traits non caractéristiques du type alpin.

Un troisième élément à affinités arménoïdes caractérise moins de $10 \%$ des sujets ; il se signale par une stature moyenne à élevée, une brachycéphalie plus ou moins accentuée et une face allongée. 

d'individus méso- ou dolichocéphales, à face basse et de stature élevée, et dont le rapport céphalo-facial rappelle celui du type de Mechta-Afalou des anciens habitants d'Afrique du nord à l'époque épipaléolithique. Les signes de métissage avec un élément négroïde (peau basanée, nez plus large, lèvres plus épaisses, cheveux frisés à crépus) sont rares dans la série étudiée et paraissent se manifester isolément, dissociation qui pourrait indiquer un apport ancien de l'élément noir plutôt que récent. Mais on ne peut exclure aussi l'hypothèse que certains de ces traits, notamment la forme frisée de la chevelure fréquente chez les Algériens, ou un certain prognatisme alvéolaire, correspondraient plutôt à des caractères adaptatifs propres aux populations méditerranéennes du littoral nord-africain et indépendantes de toute influence négroïde.

Tableau I - Distance entre les groupes sédentaires d'Algérie. (Distance générale de Hiernaux) traduisant par une pigmentation claire de la peau et des yeux ainsi qu'un blondisme de la chevelure n'apparaît pas évidente. Si la coloration de la peau est effectivement plutôt claire chez la majorité des sujets, une teinte sombre des cheveux est largement prédominante. Une proportion infime de sujets présente néanmoins une teinte châtain ou blond foncé, un peu moins rare dans les montagnes que dans les plaines, mais elle n'est pas associée, ou exceptionnellement, à une pigmentation claire de l'œil. Signalons cependant que le blondisme est fréquent chez les enfants, en Grande Kabylie notamment, comme dans la plupart des populations de la Méditerranée occidentale, caractère disparaissant généralement après l'adolescence. Les teintes sombres prédominent également dans la couleur des yeux, mais les yeux mixtes sont relativement fréquents surtout dans les régions de montagnes et les Hautes Plaines orientales ainsi que dans l'Aurès. Cette fréquence relative d'yeux mêlés n'est pas propre aux Algériens mais se rencontre dans d'autres pays d'Afrique du nord, notamment au Maroc, et se rapproche également des taux observés chez certaines populations de la Méditerranée occidentale (Italie du sud, Sicile, Corse, Sardaigne, Espagne), différente de ceux sensiblement plus bas rencontrés dans la partie orientale du Bassin méditerranéen. La fréquence des yeux clairs, quant à elle, varie entre 2 et $15 \%$ selon les régions. 
profondeur et en largeur, des membres supérieurs plutôt courts relativement à la stature, des membres inférieurs métrio- à macroskèles.

Quelques différences ont été notées entre les groupes des montagnes et ceux des plaines : tendance chez les premiers à une stature moins élevée, à l'allongement du tronc et l'élargissement des épaules, plus grande fréquence de troncs rectangulaires et thorax plus développés, tous traits étant probablement de nature adaptative.

61 Au niveau de la tête, la principale caractéristique est l'existence d'une diversité dans le rapport céphalo-facial et notamment d'une dysharmonie modérée ou accentuée chez près de la moitié des Algériens étudiés. Ceux-ci comprennent en effet entre autres plus de $30 \%$ de mésocéphales à face longue et très longue, les dolichocéphales à face longue et surtout très longue étant cependant davantage représentés dans la population. Les faces plus courtes ne sont cependant pas rares: un quart des sujets a une face de hauteur moyenne associée généralement à une tête mésocéphale. Une seconde particularité à souligner est le développement latéral fréquent des mâchoires chez les Algériens qui donne à la face un contour quadrangulaire: 30 à $50 \%$ des sujets présentent cette caractéristique, plus fréquente semble-t-il en Grande Kabylie et dans l'ouest de l'Algérie que dans les autres régions. Le nez est franchement leptorhinien, au profil généralement droit (51\%) ou convexe (39\%), le premier type étant plus fréquent dans les régions de montagne, le second étant plus représenté dans les régions de plaines. Les lèvres sont d'épaisseur moyenne, la forme des cheveux est variable, du type droit à frisé, ce dernier représentant la forme la plus fréquente dans toutes les régions, sauf chez les montagnards de l'ouest.

L'étude des processus de sénescence a montré que, comparativement à des Français appartenant à un milieu socio-professionnel analogue et vivant sous le même climat, les modalités du vieillissement sont généralement peu différentes. Cependant les manifestations de sénescence apparaissent plus tôt chez les Algériens, l'écart pouvant être estimé à une dizaine d'années, avec une rupture de pente apparaissant entre 50 et 60 ans chez eux au lieu de 60-70 chez les Français.

Des signes de changements diachroniques ont été constatés chez des séries originaires de Tizi-Ouzou et de Lakhdaria étudiées les unes à environ 30 années d'intervalle, les secondes à un siècle d'intervalle. Ces processus de micro-évolution sont analogues à ceux que l'on a constatés chez d'autres populations, c'est-à-dire augmentation de la stature, diminution de l'indice céphalique, foncement de la chevelure. Cependant comparativement aux populations européennes, ils semblent avoir été beaucoup moins marqués en Algérie, tout au moins dans les deux régions qui ont été comparées.

L'étude des dermatoglyphes digitaux d'une série importante d'Algériens originaires de la plupart des régions situées au nord du Sahara, a montré une grande homogénéité dans la distribution régionale des fréquences des arcs, des boucles et des tourbillons. Dans l'ensemble, les Algériens sont caractérisés par une faible fréquence des arcs, un taux élevé de boucles et une fréquence moyenne de tourbillons. 
Tableau II. Principales mesures des Algériens du nord actuels (d'après Chamla, 1974)

I. Corps (en $\mathrm{cm})$

\begin{tabular}{|l|c|c|c|c|c|}
\hline Mesures & N & M & E.T. & Min. & Max. \\
\hline Stature & 462 & 168,6 & 5,64 & 154,5 & 185,6 \\
Largeur épaules & 432 & 38,2 & 1,88 & 32,3 & 43,5 \\
Largeur bassin & 415 & 28,1 & 1,76 & 23,2 & 36,0 \\
Périmètre thorax & 438 & 96,3 & 5,64 & 83 & 114,5 \\
Longueur membre inf. & 281 & 95,5 & 4,32 & 85,1 & 109,9 \\
\hline
\end{tabular}

II. Tête (en mm)

\begin{tabular}{|l|c|r|c|c|c|}
\hline Longueur tête & 463 & 192,2 & 6,24 & 170 & 211 \\
Largeur tête & 463 & 147,7 & 5,55 & 132 & 164 \\
Indice céphalique & 463 & 76,7 & 3,60 & 67,7 & 87,7 \\
Longueur face & 462 & 125,9 & 6,36 & 108 & 143 \\
Largeur face & 463 & 137,4 & 5,16 & 117 & 149 \\
Indice facial morphol. & 462 & 91,6 & 5,32 & 78,2 & 107,6 \\
Largeur front & 463 & 107,7 & 4,68 & 91 & 122 \\
Largeur mâchoire & 459 & 106,2 & 5,82 & 91 & 127 \\
Hauteur nez & 461 & 54,4 & 4,24 & 40 & 69 \\
Largeur nez & 460 & 35,7 & 2,5 & 27 & 43 \\
Indice nasal & 460 & 65,6 & 7,11 & 50 & 95,5 \\
\hline
\end{tabular}

III. Caractères morphologiques (en \%)

\begin{tabular}{|llr|llr|}
\hline Forme du profil & droit & 51,8 & Couleur peau & blanche & moy. colorée \\
nasal & convexe & 39,5 & & 34,7 \\
$\mathrm{~N}=374$ & sinueux & 5,3 & $\mathrm{~N}=455$ & basanée & 10,3 \\
& concave & 3,2 & & & \\
Forme des lèvres & minces & 23,7 & Couleur cheveux & blond foncé & 0,8 \\
$\mathrm{~N}=375$ & moyennes & 45,3 & $\mathrm{~N}=450$ & châtains & 0,8 \\
& un peu êpais. & 19,7 & & brun-noirs & 23,1 \\
& épaisses & 11,2 & & noirs & 74,4 \\
& & & & roux & 0,6 \\
Forme des cheveuxlisses & 21 & Couleur yeux & bleus & 1,7 \\
$\mathrm{~N}=375$ & ondulés & 34,6 & $\mathrm{~N}=462$ & gris & 2,5 \\
& frisés & 44 & & verts & 4,5 \\
& crépus & 0,2 & & marron clair & 18,1 \\
& & & & marron foncé & 72,9 \\
\hline
\end{tabular}

\section{Dermatoglyphes digitaux}

La répartition des types de dessins digitaux, arcs, boucles et tourbillons, a été étudiée chez 2336 hommes algériens (Chamla, 1961), originaires de différentes régions. Dans l'ensemble, les Algériens sont caractérisés par une faible fréquence des arcs, des taux élevés de boucles et moyens de tourbillons. En Aurès (Chamla et Demoulin, 1979), les arcs sont plus nombreux que chez les autres groupes algériens, significatifs d'une certaine endogamie : 


\section{Pourcentages d'arcs, de boucles et de tourbillons (mains réunies, en \%)}

\begin{tabular}{|l|c|c|c|c|}
\hline & N sujets & A & B & T \\
\hline Algériens & 2336 & 3,9 & 59,1 & 36,8 \\
Aurès & 1743 & 4,5 & 58,2 & 37,2 \\
\hline
\end{tabular}

66 Comparées avec leurs voisins marocains, les proportions de ces dessins sont analogues. Comparées avec les Tunisiens, ces proportions sont également similaires, à l'exception d'un pourcentage de tourbillons plus élevé chez ces derniers.

\section{Groupes sanguins}

\section{a) - Système A B 0}

67 En Algérie septentrionale les populations berbérophones des régions montagneuses, sauf l'Aurès, sont caractérisées dans l'ensemble par un taux faible du gène $\mathrm{A}$ et élevé du gène 0 . Les habitants de l'Aurès semblent se différencier des autres régions par une fréquence plus faible de $\mathrm{A}$ et plus élevée de $\mathrm{O}$, mais les données recueillies à ce jour sur eux portent sur un nombre insuffisant de sujets et mériteraient confirmation.

Cependant, les variations phénotypiques paraissent assez grandes si l'on compare des sous-groupes régionaux constitués notamment par arrondissements. Ainsi en Kabylie occidentale, on observe des variations de A allant de $33 \%$ (L'Arba Naït Irathen, Lakhdaria) à $45 \%$ (Azazga), et des fréquences de 0 variant de $30 \%$ (Bordj Ménaïel) à 46 \% (L'Arba Naït Irathen).

69 Néanmoins, les fréquences géniques moyennes constatées chez les populations berbérophones d'Algérie septentrionale ne différent pas significativement des fréquences observées dans les autres régions de plaines et de Hautes Plaines non berbérophones. Ce qui ne saurait surprendre puisque la plupart des arabophones sont des Berbères arabisés.

70 Comparativement aux populations berbérophones du Sahara comme les Touaregs de l'Ahaggar, des Ajjer et de l'Aïr, on constate une fréquence du gène A nettement plus élevée chez les montagnards de la zone septentrionale alliée à une intensité moindre $\mathrm{du}$ gène $\mathrm{O}$. Certains groupes Touareg, notamment, se signalent par une fréquence très élevée de ce dernier gène. Le gène $B$ de son côté, paraît diminuer du Nord au Sud de l'Algérie, mais de façon moins marquée.

71 Les populations berbères de la Tunisie offrent des fréquences de $\mathrm{ABO}$ analogues à celles des habitants de la Tunisie septentrionale lesquelles sont comparables à l'ensemble des Algériens de la zone septentrionale. Les habitants de l'île de Djerba accusent cependant un taux de $\mathrm{A}$ un peu plus élevé et un taux de $\mathrm{O}$ moins fort que les autres habitants berbérophones de la Tunisie.

$72 \mathrm{Au}$ Maroc, chez les habitants du Moyen et du Haut-Atlas, la distribution est plus hétérogène et caractérisée par des variations très marquées des fréquences géniques des trois groupes, dues probablement au cloisonnement régional et à l'isolement de certains habitants de ces régions montagneuses, qui favorisent les phénomènes de dérive génique. 
73 Dans l'ensemble, on n'observe donc aucune homogénéité particulière dans les distributions des fréquences géniques du système $\mathrm{ABO}$ chez les populations berbérophones d'Afrique du nord, mais plutôt une répartition liée à l'isolement ou à l'ouverture des groupes ethniques, en particulier: $1^{\circ}$ ) une grande similitude entre l'ensemble des Kabyles et leurs voisins arabophones, ainsi qu'entre les berbérophones tunisiens et l'ensemble des Tunisiens; $2^{\circ}$ ) une augmentation du gène $\mathrm{O}$ chez les Touaregs que l'on retrouve chez l'ensemble des habitants du Sahara, mais plus marquée en raison de leur isolement; $3^{\circ}$ ) une hétérogénéité chez les Marocains des régions montagneuses due au cloisonnement régional.

\section{b) - Système Rhésus} hétérogénéité due au cloisonnement régional. Les fréquences s'établissent pour 4 groupes ethniques entre 5 et $11 \%$. Le taux serait de $21 \%$ chez les Berbères marocains en général d'après Messerlin (1950), sous réserve des erreurs de technique dans la recherche des Rh négatif à cette époque.

En Tunisie, on possède des données sur deux ethnies berbérophones de l'île de Djerba, dont les fréquences de Rhésus négatif diffèrent, cette différence étant probablement due à une absence de relation entre ces groupes. Chez les populations tunisiennes non berbérophones, on trouve un taux de Rhésus négatif analogue à celui des Algériens en général, sans différences marquées entre le nord et le sud du pays. 
Tableau III. - Fréquences phénotypiques et géniques des systèmes $A B O$, et Rhésus chez les populations berbérophones d'Afrique du nord.

SYSTĖME A B 0

\begin{tabular}{|c|c|c|c|c|c|c|c|c|c|}
\hline & \multirow{2}{*}{$\begin{array}{c}\mathrm{N} . \\
\text { sujets }\end{array}$} & \multicolumn{4}{|c|}{ Fréquences phénotypiques (\%) } & \multicolumn{3}{|c|}{ Fréquences géniques } & \multirow[t]{2}{*}{ Auteurs } \\
\hline & & A & B & $\mathrm{AB}$ & 0 & $\mathrm{p}$ & $\mathrm{q}$ & $\mathrm{r}$ & \\
\hline Algérie : & & & & & & & & & \\
\hline Kabylie occid. & 1168 & 35,9 & 17,0 & 4,7 & 42,3 & 0,230 & 0,116 & 0,654 & Benabadji, Chamla, 1971 \\
\hline Kabylie orient. & 387 & 34,6 & 16,0 & 4,6 & 44,7 & 0,221 & 0,109 & 0,670 & id. \\
\hline Chaouïas (Aurès) & 79 & 25,3 & 12,6 & 2,5 & 59,5 & 0,151 & 0,079 & 0,770 & id. \\
\hline Massifs mont. ouest & 122 & 32,8 & 16,4 & 5,7 & 45,0 & 0,216 & 0,117 & 0,667 & id. \\
\hline Touareg : Ahaggar & 57 & 8,7 & 14,0 & 1,7 & 75,4 & 0,054 & 0,082 & 0,863 & Benabadji et coll., 1965 \\
\hline Ajjer & 89 & 15,7 & 12,4 & 1,1 & 70,8 & 0,088 & 0,070 & 0,841 & Mandoul et coll., 1953 \\
\hline Aïr & 95 & 22,1 & 10,5 & - & 67,3 & 0,118 & 0,054 & 0,827 & Benabadji et coll. 1965 \\
\hline \multicolumn{10}{|l|}{ Maroc: } \\
\hline Rifains & 196 & 18,3 & 28,6 & 3,1 & 50,0 & 0.114 & 0,174 & 0,712 & Coon 1931 \\
\hline Aït Haddidou & 256 & 11,6 & 7,8 & 0,8 & 79,7 & 0,064 & 0,044 & 0,891 & Johnson et coll, 1963 \\
\hline Zaïans & 985 & 28,0 & 19,3 & 3,9 & 48,7 & 0,175 & 0,124 & 0,700 & Levêque, 1955 \\
\hline Beni Mathar & 526 & 36,3 & 20,5 & 7,9 & 35,1 & 0,253 & 0,154 & 0,592 & Levêque, 1955 \\
\hline Chaouias (Guisser) & 320 & 35,9 & 11,8 & 3,4 & 48,7 & 0,221 & 0,079 & 0,698 & Méchali, 1955 \\
\hline \multicolumn{10}{|l|}{ Tunisie : } \\
\hline Berbères Douiret & 500 & 32,4 & 15,8 & 5,4 & 46,4 & 0,211 & 0,112 & 0,677 & Caillon et Disdie, 1930 \\
\hline île Djerba & 148 & 40,5 & 12,8 & 4,7 & 42,0 & 0,260 & 0,092 & 0,647 & Gherib et coll, 1965 \\
\hline
\end{tabular}

\section{SYSTÈME RHÉSUS}

\begin{tabular}{|c|c|c|c|c|c|c|}
\hline & $\begin{array}{l}\mathrm{N} . \\
\text { sujets }\end{array}$ & \multicolumn{2}{|c|}{$\begin{array}{c}\text { Fr. phénotypiques } \\
\text { (en \%) } \\
\mathrm{Rh}+\mathrm{Rh}-\end{array}$} & \multicolumn{2}{|c|}{ Fr. géniques } & Auteurs \\
\hline Algérie: & & & & & & \\
\hline Kabylie occident. & 1168 & 92,2 & 7,8 & 0,721 & 0,279 & Benabadji et Chamla, 1971 \\
\hline Kabylie orient. & 387 & 88,3 & 11,6 & 0,659 & 0,341 & id. \\
\hline Chaouïas (Aurès) & 79 & 93,6 & 6,3 & 0,749 & 0,251 & id. \\
\hline Massifs mont. ouest & 122 & 91,8 & 8,2 & 0,714 & 0,286 & id. \\
\hline Touareg : Ahaggar & 57 & 85,9 & 14,0 & 0,623 & 0,376 & Benabadji et coll., 1965 \\
\hline Aïr & 95 & 84,4 & 15,5 & 0,604 & 0,395 & id. \\
\hline \multicolumn{7}{|l|}{ Maroc: } \\
\hline Berbères en génér. & 987 & 78,6 & 21,4 & 0,538 & 0,462 & Messerlin 1950 in Mourant 1958 \\
\hline Aït Haddidou & 256 & 94,5 & 5,4 & 0,766 & 0,234 & Johnson et coll., 1963 \\
\hline Zaians & 630 & 88,5 & 11,4 & 0,662 & 0,338 & Levêque 1955 \\
\hline Chaouïas (Guisser) & 320 & 90,9 & 9,0 & 0,699 & 0,301 & Méchali, 1955 in Mourant, 1970 \\
\hline Beni Mathar & 473 & 94,3 & 5,7 & 0,761 & 0,239 & Levêque, 1955 \\
\hline \multirow{2}{*}{\multicolumn{7}{|c|}{$\begin{array}{l}\text { Tunisie : } \\
\text { île Djerba : }\end{array}$}} \\
\hline & & & & & & \\
\hline Wahabites & 329 & 91,8 & 8,2 & 0,713 & 0,286 & Ranque et coll., 1961 \\
\hline Malekites & 258 & 87,2 & 12,8 & 0,642 & 0,357 & id. \\
\hline
\end{tabular}

\section{Position anthropologique des Algériens vis-à-vis des populations du nord de l'Afrique et du Bassin méditerranéen}

La position anthropologique des Algériens par rapport aux autres populations araboberbères de l'Afrique septentrionale, inclus le Sahara, et dont l'étude a été faite par la méthode de la distance générale de Hiernaux basée sur les moyennes des caractères 
métriques, les pourcentages des boucles et des tourbillons pour les dermatoglyphes, et les fréquences géniques des groupes $\mathrm{ABO}$ et $\mathrm{Rh}$, indique des affinités certaines entre les Libyens et les Algériens. Ceux-ci se rapprochent également des Marocains, mais offrent moins de similitude avec les Tunisiens et les Égyptiens. Un second fait intéressant est l'éloignement marqué qui existe entre les Algériens de la région septentrionale - et d'une façon générale les groupes sédentaires du nord de l'Afrique - et les habitants nomades du Sahara. La comparaison des groupes nomades indique par ailleurs une diversité manifeste, montrant qu'il existe plus de divergence entre eux qu'entre les groupes sédentaires du nord, même éloignés géographiquement. Ces divergences entre les Algériens de la zone septentrionale et les nomades sahariens montrent que le Sahara a joué le rôle d'une barrière anthropologique, isolant en outre les unes des autres les populations qui l'habitent, facteur favorable à l'apparition de caractères biologiques spécifiques.

Profils graphiques de populations d'Afrique septentrionale par référence aux Algériens.
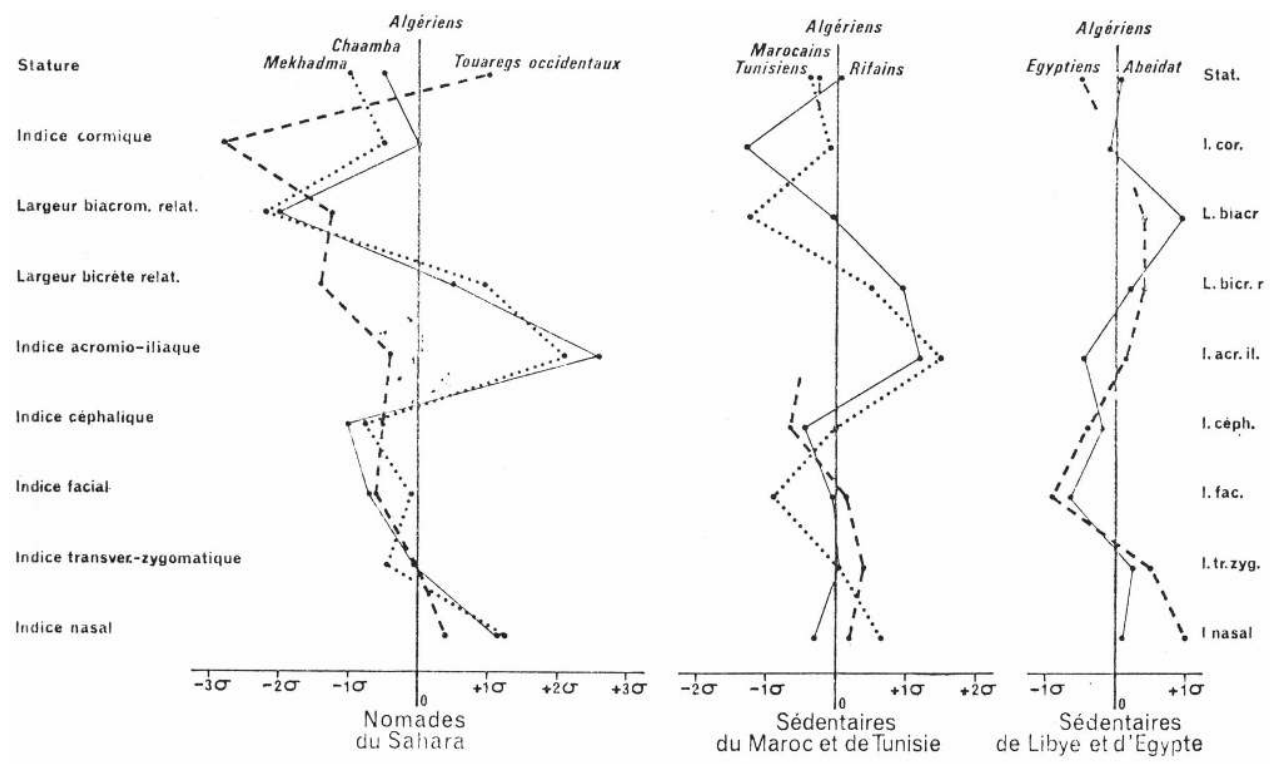
Distance $\Delta \mathrm{g}$ entre les Algériens et les populations sédentaires et nomades du Bassin méditerranéen et du Sahara.

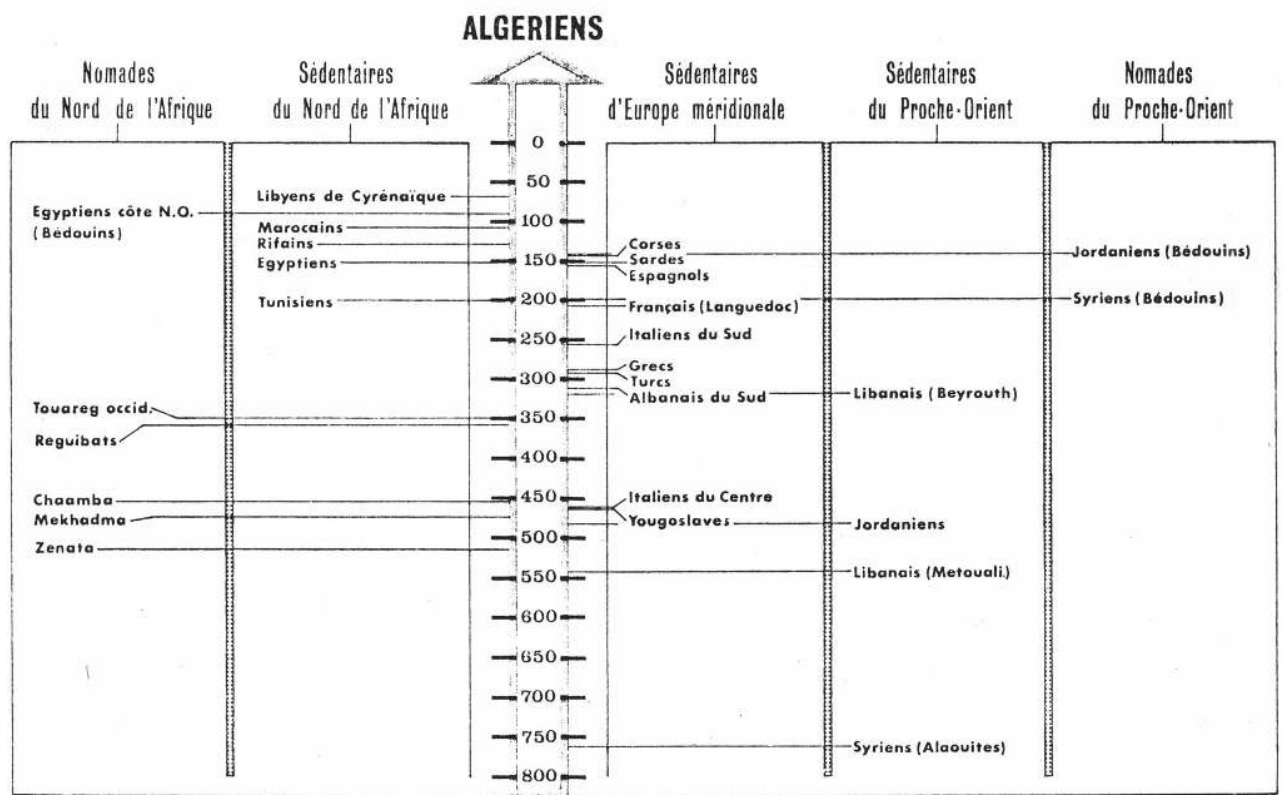

79 Au total il semble exister moins de divergences entre les groupes sédentaires, même éloignés géographiquement, que parmi les groupes nomades sahariens. Les coefficients de distance entre tous les groupes sédentaires varient de 67 à 254 . Ceux des groupes nomades s'échelonnent entre 56 et 534 . On ne peut guère attribuer a priori à des processus adaptatifs cette disparité entre les groupes nomades, puisqu'ils vivent dans un milieu analogue et dans des conditions de vie probablement peu différentes. Peut-on l'expliquer par une origine différente, hypothèse qui parait peu probable mais difficile à vérifier? Plus vraisemblablement, mais les données anthropologiques à notre disposition sont alors insuffisantes pour répondre avec certitude, il s'agit là de phénomènes de dérive génétique auxquels s'ajoutent peut-être des facteurs de consanguinité qui pourraient être à l'origine d'une diversification des caractères biologiques de ces groupes.

Tableau IV - Distance entre les populations sédentaires et nomades du Nord de l'Afrique ( $\Delta \mathrm{g}$ de Hiernaux)

SEDENTAIRES

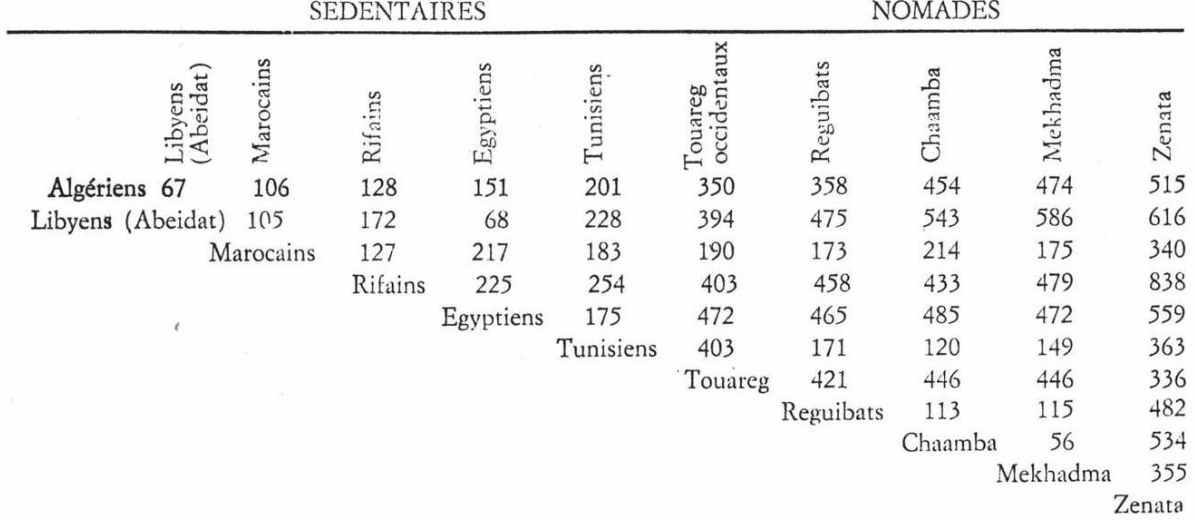


Tableau V - Distance $\Delta \mathrm{g}$ entre nomades du Sahara, Bedouins d'Égypte et du Proche-Orient

\begin{tabular}{|c|c|c|c|c|c|}
\hline \multicolumn{2}{|c|}{ Touareg occ. } & Reguibats & Zenata & Béd. Jordaniens & Béd. Syriens \\
\hline \multirow[t]{6}{*}{ Bédouins d'Egypte } & 303 & 347 & 430 & 138 & 137 \\
\hline & Touareg & 421 & 336 & 306 & 363 \\
\hline & & Reguibats & 482 & 241 & 217 \\
\hline & & & Zenata & $(1073)(1)$ & $(469)(1)$ \\
\hline & & & & Bédouins Jordani & ns 200 \\
\hline & & & & Bé & ouins Syriens \\
\hline
\end{tabular}

80 La position anthropologique des Algériens vis-à-vis des autres populations du Bassin méditerranéen, analysée également par la méthode de la distance générale de Hiernaux, a montré qu'il existe des affinités entre eux et certaines populations de l'Ouest méditerranéen comme les Corses, les Sardes, les Espagnols, les Italiens du sud. En revanche, les coefficients de distance sont apparus particulièrement élevés entre les Algériens et les Italiens du Centre, ainsi que les Yougoslaves et les populations sédentaires du Proche-Orient comme les Jordaniens, les Libanais et les Syriens. Chez ces populations, le type arménoïde très différent du type méditerranéen, est en effet largement représenté, alors qu'il est peu répandu en Algérie. Il n'y a donc pas lieu de s'étonner de ces divergences. Par contre, la distance entre certains Bédouins du Proche-Orient où prédomine le type méditerranéen, et les Algériens, est apparue beaucoup moins marquée.

81 L'origine des affinités entre les Algériens et les populations de l'Ouest méditerranéen ne peut s'expliquer sur la seule base des données biologiques actuelles, mais doit être recherchée dans le passé anthropologique de la population algérienne ainsi que dans les données historiques et archéologiques à notre disposition. Ces dernières pour leur part semblent démontrer l'existence de courants culturels dès l'époque protohistorique et peut-être même remontant à une époque antérieure, entre le Maghreb et des pays de la Méditerranée centrale. Si ces échanges ont été accompagnés de migrations, on doit pouvoir en retrouver les traces dans les restes humains datant de l'époque protohistorique qui ont été recueillis dans de nombreux sites d'Afrique du nord.

La probabilité de l'existence d'échanges culturels entre l'Europe protohistorique et le Maghreb a été démontrée ailleurs. En effet selon G. Camps (1970) : « La répartition de certains types très caractéristiques de monuments funéraires protohistoriques tels que les dolmens ou les hypogées cubiques de Tunisie du nord, la limitation aux seules zones méditerranéennes de la poterie peinte... et bien d'autres faits secondaires sont autant de témoins indiscutables d'une pénétration non seulement de courants culturels mais de groupes humains venus des pays et des îles de la Méditerranée centrale avant les temps historiques $»$.

83 Mais ces échanges culturels ont-ils été accompagnés réellement de migrations suffisamment importantes pour modifier la composition de la population qui habitait l'Algérie antérieurement à cette époque ? Une réponse à une telle question ne pourrait être fournie que par l'étude diachronique de l'évolution du peuplement de l'Algérie depuis les temps préhistoriques, basée sur des documents ostéologiques en nombre suffisant. Une telle étude avait été entreprise par Bertholon et Chantre en 1913, mais les documents de base avaient été insuffisamment exploités. Depuis cette époque, de nombreuses fouilles ont mis au jour un matériel ostéologique humain important datant de diverses périodes, qui fournit des éléments d'appréciation suffisants pour tenter 
d'élucider ce problème de l'origine du peuplement de l'Algérie. D'ores et déjà, la position anthropologique des Algériens vis-à-vis d'une part des Libyens et des Bédouins du Proche-Orient, de l'autre de certaines populations de la Méditerranée occidentale, fournit des indications intéressantes permettant d'orienter les recherches ultérieures.

Tableau VI Populations du Bassin méditerranéen. Références bibliographiques.

\begin{tabular}{|c|c|c|c|c|}
\hline \multirow{2}{*}{ Populations } & \multirow{2}{*}{$\begin{array}{l}\text { Caractères } \\
\text { métriques }\end{array}$} & \multicolumn{2}{|c|}{ Groupes sanguins } & \multirow{2}{*}{ Dermatoglyphes } \\
\hline & & $\mathrm{ABO}$ & $\mathbf{R h}$ & \\
\hline Espagn. (Aragonais)... & $\begin{array}{c}\text { Fusté et Pons, } \\
1062 .\end{array}$ & $\begin{array}{c}\text { Hoyos Sainz, } \\
1947 .\end{array}$ & Guaseh, 1952. & $\begin{array}{l}\text { Oloriz, } 19088 \\
\text { (Espagnols } \\
\text { en gal). }\end{array}$ \\
\hline $\begin{array}{l}\text { Franc. du Languedoc } \\
\text { Corses ............... }\end{array}$ & $\begin{array}{c}\text { Olivier, } 1970 . \\
\text { Piquet-Thépot, } \\
1963 .\end{array}$ & $\begin{array}{c}\text { Olivier, id. } \\
\text { Olivier, } 1970 .\end{array}$ & $\begin{array}{l}\text { Olivier, id. } \\
\text { Nicoli et coll., } \\
1959 .\end{array}$ & $\begin{array}{c}\text { Olivier, id. } \\
\text { Piquet-Thépot, } \\
1963\end{array}$ \\
\hline $\begin{array}{r}\text { Italiens du Centre } \\
\text { (Pérouse) }\end{array}$ & Sorcetti, 1964. & Sorcetti, id. & Sorcetti, id. & \\
\hline $\begin{array}{r}\text { Italiens du Sud (Ro- } \\
\text { frano, Salerne) }\end{array}$ & Cresta, 1958. & Cresta, id. & $\begin{array}{l}\text { Passarello, } \\
1958 .\end{array}$ & Falco, 1908. \\
\hline Sardes .. & $\begin{array}{c}\text { Jucei } \\
\text { (in Biasutti). }\end{array}$ & Lattes, 1932. & Morganti, 1949. & $\begin{array}{c}\text { Vrydagh- } \\
\text { Laoureux, } 1966 .\end{array}$ \\
\hline Yougoslaves $\ldots \ldots \ldots$ & Jovanovic, 1958. & $\begin{array}{c}\text { Kalic } \\
\text { et Kostic, } 1934\end{array}$ & $\begin{array}{c}\text { Simonovic, } \\
1953 .\end{array}$ & $\begin{array}{c}\text { Abel, } 1940 \\
\text { (in Schwidetsky). }\end{array}$ \\
\hline Grees .. & Hertzberg, 1963. & $\begin{array}{c}\text { Papageorgiu, } \\
1952 .\end{array}$ & $\begin{array}{l}\text { Papageorgiu, } \\
\text { id. }\end{array}$ & $\begin{array}{c}\text { Roberts et coll., } \\
1965 .\end{array}$ \\
\hline Albanais du Sud .... & Tildesley, 1963. & $\begin{array}{l}\text { Simonovic, } \\
1953 .\end{array}$ & - & - \\
\hline Tures ....... & Hertzberg, 1963. & Onur, 1937. & - & $\underset{\text { (in Schwinalev }}{\text { Engietsky). }}$ \\
\hline $\begin{array}{l}\text { Libanais (Metouali).. } \\
\text { Libanais sédent. .... }\end{array}$ & $\begin{array}{l}\text { Shanklin, } 1938 . \\
\text { Seltzer, } 1936 .\end{array}$ & $\begin{array}{c}\text { Giraud-Costa, } \\
1952 . \\
\text { Taleb, } 1964 .\end{array}$ & $\begin{array}{c}- \\
\text { Taleb, id. }\end{array}$ & $\begin{array}{c}\text { Cummins et } \\
\text { Shanklin, 1937. } \\
\text { Shanklin et } \\
\text { Cummins, 1937. }\end{array}$ \\
\hline $\begin{array}{c}\text { Syriens sédent. (Ala- } \\
\text { ouites) }\end{array}$ & $\begin{array}{c}\text { Vallois } \\
\text { et Chamla, } 1964 .\end{array}$ & $\begin{array}{l}\text { Altounyan, } \\
1928 .\end{array}$ & - & - \\
\hline Syriens béd. (Rouala). & Shanklin, 1935. & $\begin{array}{c}\text { Boyd et Boyd, } \\
1938 .\end{array}$ & - & $\begin{array}{c}\text { Shanklin et } \\
\text { Cummins, } 1937 .\end{array}$ \\
\hline Jordan & Gloor, 1950. & $\begin{array}{c}\text { Taleb } \\
\text { et Ruffié, } 1968 .\end{array}$ & $\begin{array}{c}\text { Taleb } \\
\text { et Ruffié, id. }\end{array}$ & - \\
\hline Jordaniens bédouins & $\begin{array}{l}\text { Vallois, } 1959 . \\
\text { (Taamré). }\end{array}$ & $\begin{array}{c}\text { Taleb } \\
\text { et Ruffié, } 1968 .\end{array}$ & $\begin{array}{l}\text { Taleb } \\
\text { et Ruffié, id. }\end{array}$ & - \\
\hline $\begin{array}{r}\text { Egyptiens bédouins } \\
\text { (Sidi Barani et So- } \\
\text { loum) } \ldots \ldots \ldots \ldots \ldots \ldots\end{array}$ & $\begin{array}{c}\text { Godycki et } \\
\text { Dzierzykray, } \\
1964 .\end{array}$ & $\begin{array}{c}\text { Godycki et } \\
\text { Dzierzykray, } \\
\text { id. }\end{array}$ & - & - \\
\hline
\end{tabular}

Tableau VII Distance $\Delta \mathrm{g}$ des Algériens vis-à-vis des populations sédentaires et nomades du Bassin méditerranéen (Europe, Proche-Orient, Égypte).

\begin{tabular}{|c|c|c|c|}
\hline Algériens & & & \multirow{10}{*}{ Europe. } \\
\hline Aigeriens & Siardes. & 143 & \\
\hline 》 & Espagnols & $\begin{array}{l}151 \\
156\end{array}$ & \\
\hline$》$ & Français (Languedoc) & 207 & \\
\hline$\gg$ & Italiens du Sud $\ldots \ldots \ldots \ldots \ldots \ldots$ & 256 & \\
\hline$\gg$ & Grees $\ldots \ldots \ldots \ldots \ldots \ldots \ldots \ldots$ & 288 & \\
\hline$\gg$ & $\ldots \ldots \ldots \ldots \ldots \ldots \ldots$ & 292 & \\
\hline$\gg$ & 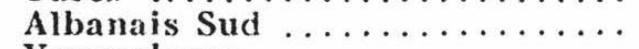 & 311 & \\
\hline$\gg$ & Yougoslaves $\ldots \ldots \ldots \ldots \ldots \ldots$ & 461 & \\
\hline 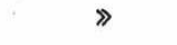 & Italiens du Centre $\ldots \ldots \ldots \ldots$ & 463 & \\
\hline$\gg$ & Jordaniens bédouins .. & 141 & \multirow{6}{*}{ Proche-Orient } \\
\hline » & Syriens bedouins $\ldots \ldots \ldots \ldots \ldots$ & 198 & \\
\hline » & Libanais sédent. ............. & 318 & \\
\hline$»$ & Jordaniens sédent. $\ldots \ldots \ldots \ldots \ldots$ & 483 & \\
\hline$\gg$ & Libanais sed. (Metouali) & 542 & \\
\hline$\gg$ & Syriens sédent. (Alaouites) $\ldots \ldots$ & 762 & \\
\hline$\gg$ & $\begin{array}{l}\text { Egyptiens bédouins (côte Nord- } \\
\text { Owest) }\end{array}$ & & \multirow{2}{*}{ V!t!m/r. } \\
\hline$\gg$ & Ligyptiens sedentaires . . . . . . & 151 & \\
\hline
\end{tabular}




\section{Conclusion}

84 résultant de l'étude de séries d'Algériens originaires de différents régions du Tell et du littoral, montrent qu'il existe un brassage particulièrement intense dans la population algérienne. Les distances générales $\Delta g$ de Hiernaux entre les différents groupes régionaux sont en effet très faibles. Cependant, l'analyse typologique montre qu'il existe des éléments morphologiques variés en proportions sensiblement équivalentes dans les régions étudiées. Les Méditerranéens représentent une large majorité, groupant 71 à $84 \%$ des sujets. Parmi eux, les Atlanto-Méditerranéens mésocéphales paraissent prédominer sur les Méditerranéens dolichocéphales. La fréquence des premiers s'accroît vers l'Ouest. Un élément brachycéphale est également présent dans une proportion d'environ $10 \%$, mais il ne peut être assimilé au type alpin européen et semblerait correspondre à des Méditerranéens brachycéphalisés. Le type arménoïde se rencontre chez moins de $10 \%$ de la population. Une fréquence très faible d'individus présente des traits de l'ancien type de Mechta-Afalou.

Crânes masculins actuels : 1 et 2 de Kabylie ; 3 de la région d'Oran.

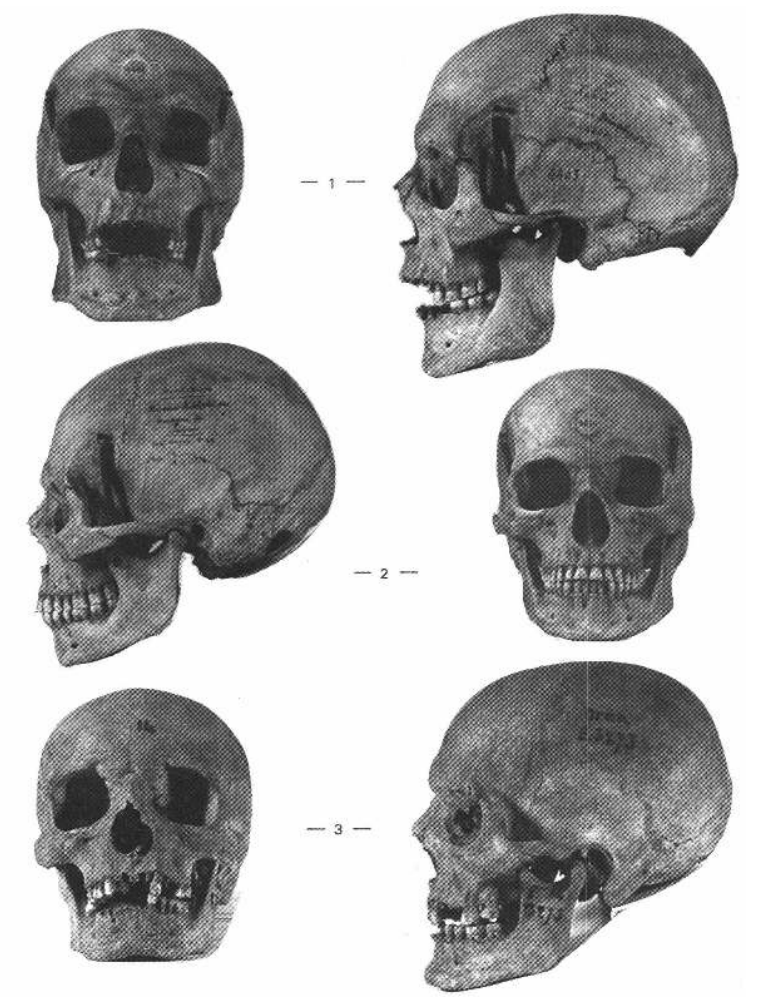

85

Les distances entre les populations sédentaires d'Afrique septentrionale apparaissent plus faibles que celles existant entre les Algériens sédentaires et les groupes nomades du Sahara. Parmi les premières, ce sont les Libyens qui paraissent être les plus proches des Algériens. La comparaison des séries nomades sahariennes entre elles donne des coefficients variables indiquant une diversité manifeste.

La position anthropologique des Algériens du Tell par rapport aux autres populations du Bassin méditerranéen montre qu'ils se rapprochent davantage des méditerranéens occidentaux (Corses, Sardes, Espagnols) que des habitants du Sud-est de l'Europe 
(Yougoslaves, Albanais, Grecs) ou des groupes sédentaires du Proche-Orient (Libanais, Jordaniens, Syriens).

\section{Anthropologie physique des Tunisiens (M.-C. Chamla)}

Deux études seulement ont été publiées sur l'anthropologie morphologique des Tunisiens; elles sont anciennes : 1133 hommes mesurés par Collignon (1887), âgés de 21 à 30 ans ; 136 Tunisiens mesurés par Marie et Mc Auliffe (1930). En revanche, entre 1930 et 1965, ont paru quelques études sur les groupes sanguins. Les dermatoglyphes digito-palmaires ont été étudiés par Chamla et Sahly (1972 et 1973).

\section{Caractères métriques et descriptifs}

88 Chez les Tunisiens mesurés par Collignon à la fin du siècle dernier, la moyenne générale de la stature n'était pas élevée $(166 \mathrm{~cm})$, correspondant à une taille surmoyenne, avec des îlots de tailles sous-moyennes dans le Nord-ouest et de tailles élevées chez les nomades du Sud. Elle semble être plus élevée vers 1920 (168 cm), d'après Marie et Mc Auliffe, mais l'échantillon est restreint.

89 La largeur du bassin est relativement étroite par rapport à celle des épaules. Cependant, l'indice acromio-iliaque (bassin/épaules) indique un tronc de forme rectangulaire. On n'a pas de données sur la longueur du membre inférieur, mais le rapport de taille assis/stature, indique un indice cormique de 52 à 53, traduisant un buste de hauteur moyenne.

90 La longueur de la tête est de $191 \mathrm{~mm}$ (C.) et $193 \mathrm{~mm}$ (M. et M.) en moyenne. La largeur, de 145 et $146 \mathrm{~mm}$, correspond à une tête longue et relativement étroite, donnant un indice céphalique en moyenne mésocéphale à la limite de la dolichocéphalie.

91 La hauteur de la face n'est pas très grande, et sa largeur moyenne donnent un indice facial morphologique de 87, soit une face moyennement longue, contrastant avec les faces plutôt longues de leur voisins Algériens. Le front est large par rapport à la face. La hauteur du nez est de $49 \mathrm{~mm}$ pour une largeur de $35 \mathrm{~mm}$, soit un indice nasal de 70,2, correspondant à un nez moyennement large.

On ne possède pas de données sur la forme du profil nasal ni sur celle des cheveux. Pour la couleur des cheveux, les teintes foncées sont prédominantes (93\%). Il n'y a pas de roux. Les yeux sont en majorité marron foncé, $20 \%$ ont des yeux de couleur mixte, $3,5 \%$ des yeux clairs. Les types de coloration (formule de Livi) montrent une majorité de types foncés $(84 \%)$.

\section{Dermatoglyphes digito-palmaires}

93 Les empreintes digitales et palmaires des Tunisiens ont été étudiées (Emberger et al., 1970 ; Chamla et Sahly, 1972 et 1973). Les secondes portent sur 1852 hommes et 241 femmes des 13 gouvernorats de Tunisie. Dans l'ensemble, les Tunisiens des régions centre et sud paraissent se rapprocher davantage des Libyens et des Égyptiens que des Algériens du point de vue de la fréquence des boucles et des tourbillons, alors que les séries du nord sont comparables à leurs voisins occidentaux. Dans l'ensemble, la fréquence des tourbillons augmente de l'Ouest à l'Est de l'Afrique septentrionale et 
celle des boucles diminue (Chamla 1962 et 1963). La terminaison de la ligne palmaire D et du type 11 pour la main droite. A la main gauche, un nombre plus élevé du type 9 et du type 7 est à noter.

\section{Groupes sanguins $A B O$ et système Rhésus}

Quatre études ont été publiées sur le système ABO. Chez les Berbères de Tunisie en général (Caillon et Disdier 1931), on observe une majorité de sujets O (46\%), de même que chez les Tunisiens en général (Ghérib, 1965). La fréquence du groupe $O$ augmente du nord au sud ( $56 \%$ ). Chez les Douiret, $58 \%$. Dans l'île de Djerba où domine l'élément berbère, en revanche, on observe une similitude entre les fréquences des types A (40 \%) et 0 ( $42 \%$ ) (effet d'endogamie ?).

Une seule étude a paru sur le système Rhésus (Ranque et al., 1961). Les sujets Rh positifs sont largement prédominants et les négatifs oscillent entre 7 et $8 \%$, correspondant donc à une moindre fréquence que chez les Algériens (7 à $17 \%$ ).

On ne possède pas de données sur les hémoglobinopathies, sur les thalassémies, ni sur les défauts en G-6-P.D.

\section{Position anthropologique des Tunisiens}

La formule de la distance générale de Hiernaux a été utilisée pour les Tunisiens (fig. 1). Ils se classent entre les Égyptiens et les Touaregs occidentaux et sont loin des Algériens et des Libyens.

Distance entre les populations sédentaires et nomades du Nord de l'Afrique ( $\Delta \mathrm{g}$ de Hiernaux)

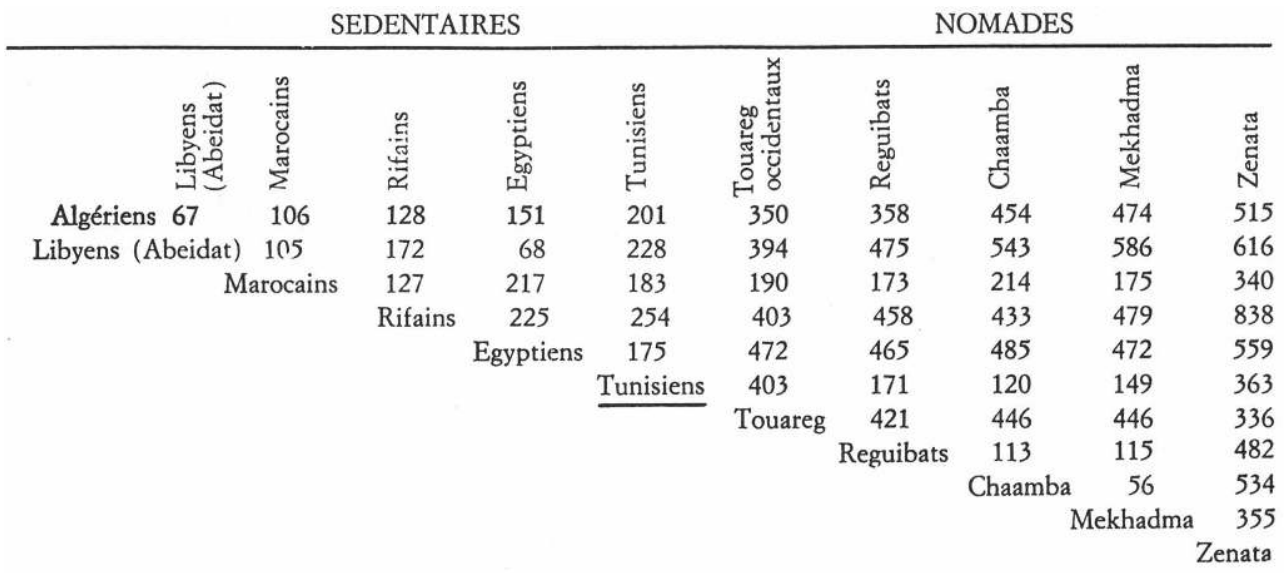

\section{Anthropologie physique des Libyens (M.-C. Chamla)}

Trois études ont paru sur la morphologie des populations libyennes du nord: deux de Puccioni (1934) sur un groupe d'El Abeidat de la Cyrénaïque septentrionale et sur un groupe d'El Baraghit de la côte nord-ouest. En Tripolitaine du nord, 198 hommes ont été étudiés par Bertholon et Chantre (1913). 


\section{Caractères morphologiques et descriptifs}

La stature est sur-moyenne chez les trois populations, 165 à $169 \mathrm{~cm}$. Les épaules sont larges $(39 \mathrm{~cm})$ par rapport au bassin $(27,7 \mathrm{~cm})$ chez les Abeidat dont l'indice acromioiliaque (rapport largeur bassin/largeur épaules) est de 71,5 en moyenne, indiquant un tronc intermédiaire à la limite du trapézoïdal. Le périmètre thoracique est de $86 \mathrm{~cm}$ en moyenne, donc relativement faible. La hauteur du buste est de $52,5 \mathrm{~cm}$ chez cette population, métriocorme. On ne possède pas de donnée sur les deux autres populations.

L'indice céphalique en Tripolitaine est de 75,4, chez les Abeidat et de 76,1 et 76,7 chez les El Baraghit, indiquant une mésocéphalie. La hauteur de la face est moyenne (120,4 $\mathrm{mm}$ ) chez les Abeidat, sa largeur est également moyenne, 137,2 mm, donnant un indice facial morphologique de 88 , assez élevé, bien qu'à la limite de la mésoprosopie. Les dimensions du nez, 54,5 $\mathrm{mm}$ pour la hauteur chez les Abeidat, $35,7 \mathrm{~mm}$ pour la largeur, correspondent à un indice nasal de 66,5, étroit. La forme du profil nasal est droite $(56 \%)$ à convexe $(41 \%) ; 3 \%$ ont un profil concave chez les Abeidat. Chez les El Baraghit, profils droit, $52,1 \%$, convexe $42,8 \%$, concave, $4,9 \%$. L'épaisseur des lèvres, mince à moyenne chez les Abeidat $(49,5 \%$ et $40,4 \%)$; mince $(66,4 \%)$ chez les El Baraghit qui ont 31,6\% de lèvres moyennement épaisses. La forme des cheveux est lisse (27\%) chez les Abeidat, 32,6\% chez les El Baraghit; ondulée, $67,4 \%$ et $66 \%$ respectivement ; frisée, $5,1 \%$ chez les Abeidat, $1,3 \%$ de cheveux crépus chez les El Baraghit. La couleur des cheveux est $100 \%$ noire chez les Abeidat. Celle des yeux, $73 \%$ de marron foncé, $26 \%$ de marron clair.

\section{Dermatoglyphes digitaux}

Quatre études ont paru sur cette variable concernant des Libyens de «type arabe » et de "type berbère ", des Dauada du Fezzan et des habitants de la Tripolitaine. les résultats sont indiqués ci-dessous en $\%$.

\begin{tabular}{|l|c|c|c|c|}
\hline & $\mathrm{N}$ & Arcs & Boucles & Tourbillons \\
\hline Libyens (type arabe) & $250 \mathrm{H}$ & 3,8 & 57,6 & 38,4 \\
Libyens (type berbère) & $250 \mathrm{H}$ & 3,2 & 56,7 & 39,9 \\
Dauada (Fezzan) & $146 \mathrm{H}-181 \mathrm{~F}$ & 1,0 & 56,7 & 42,9 \\
Tripolitaine & $167 \mathrm{H}-105 \mathrm{~F}$ & 2,5 & 54,3 & 43,2 \\
\hline
\end{tabular}

102 On a donc une prédominance, partout, de la fréquence des boucles et une très faible fréquence des arcs.

\section{Groupes sanguins $A B O$}

103 Trois études ont été publiées concernant des Marabtin (Nord-est), des Abeidat de Cyrénaïque et des Touaregs du Fezzan. Les fréquences des groupes sont les suivantes :

\begin{tabular}{|l|c|c|c|c|c|c|c|c|c|}
\hline & $\mathrm{N}$ & $\mathrm{A}$ & $\mathrm{B}$ & $\mathrm{AB}$ & $\mathrm{O}$ & $\mathrm{p}$ & $\mathrm{q}$ & $\mathrm{r}$ & Auteurs \\
\hline Marabtin & 270 & 31,5 & 21,1 & 2,6 & 44,8 & .189 & .127 & .683 & Medulla, 1931 \\
Abeidat & 275 & 30,1 & 19,6 & 9,4 & 40,0 & .228 & .163 & .632 & Mazzella, 1949 \\
Touareg & 592 & 28,0 & 19,8 & 4,8 & 47,5 & .180 & .132 & .689 & Leblanc, 1946 \\
\hline
\end{tabular}


Le groupe $\mathrm{O}$ est prédominant chez les trois populations, particulièrement chez les Touaregs, indiquant une certaine consanguinité et un isolement par rapport aux autres ethnies.

\section{Position anthropologique des Libyens}

La distance $\Delta \mathrm{g}$, selon la formule de Hiernaux qui comprend les caractères métriques, les dermatoglyphes digitaux et les groupes sanguins, indique que les Abeidat sont particulièrement proches des Algériens et des Marocains, fait intéressant qu'il convient de souligner, indiquant peut-être l'existence d'un fond berbère ancien commun aux trois populations chez lesquelles l'élément arabe, arrivé à partir du vII ${ }^{\mathrm{e}}$ siècle, a exercé peu d'influence du point de vue anthropologique.

Distance entre les populations sédentaires et nomades du Nord de l'Afrique ( $\Delta \mathrm{g}$ de Hiernaux)

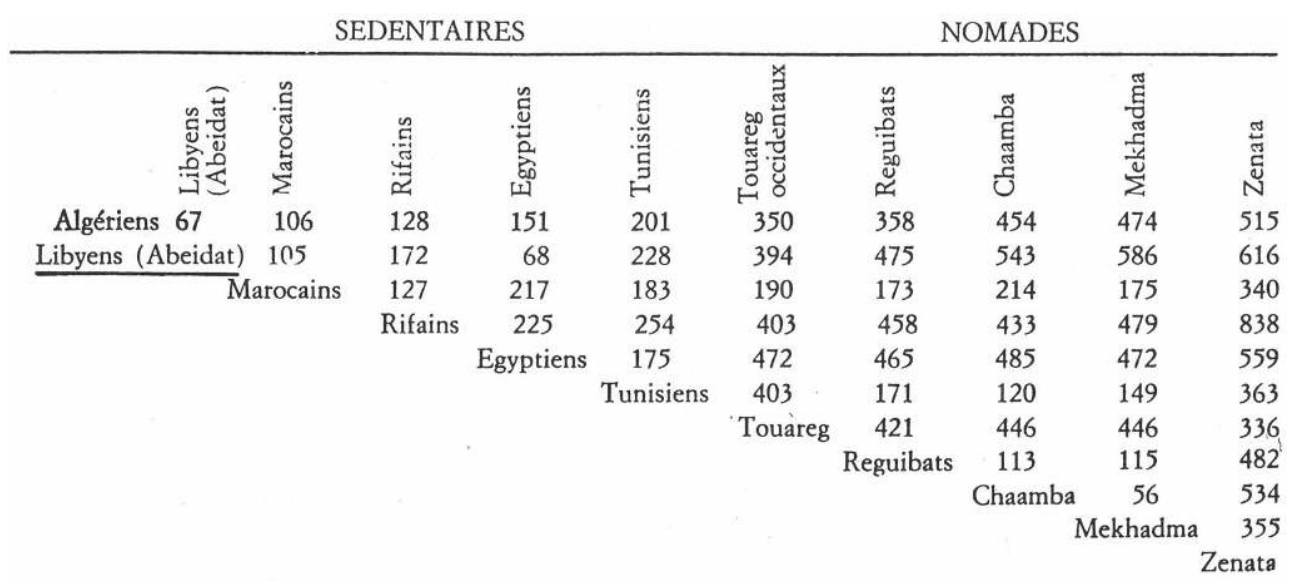

\section{Anthropologie physique des populations du Sahara (M.-C. Chamla)}

L'anthropologie morphologique des populations arabo-berbères du Sahara n'est pas connue globalement. Seules des études ponctuelles ont paru, certaines fort anciennes. Une dizaine de groupes ethniques ont été étudiés : Mozabites sédentaires (Amat, 1888, Chantre, 1910); Chaamba nomades de Ghardaïa et de Metlili (Coblentz, 1967); Mekhadma nomades de Ouargla (Coblentz, 1967); Reguibat nomades de Tindouf (Coblentz, 1967); Zenata nomades d'El Golea, Timimoun, Ain Salah (Leblanc, 1934, revus par Briggs en 1958); Mrabtines sédentaires d'Idelès (Ignazzi, 1969); Touaregs " occidentaux» nomades de l'Ahaggar, de l'Ajjer et des Ifoghas, réunis par Briggs d'après divers auteurs (1955) ; Touaregs « orientaux » originaires du Fezzan réunis par Briggs (1955) d'après des documents publiés par Chantre (1910) et Cipriani (1937).

Les groupes sanguins des habitants du Sahara (groupes ABO et système Rhésus) ont fait, en revanche, l'objet de nombreuses publications (cf la bibliographie), ainsi que la fréquence des hémoglobines anormales, thalassémies et défaut en G-6-P.D. 
Origine des échantillons des populations arabo-berbères du Sahara.

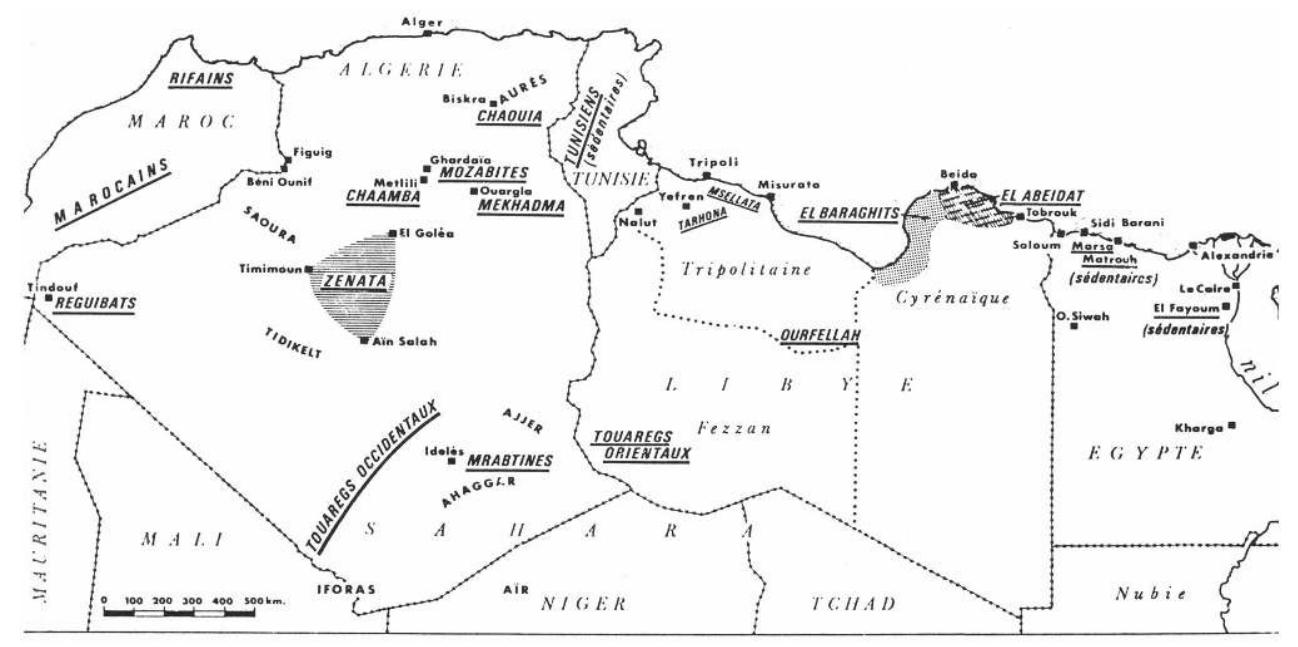

\section{Caractères métriques et descriptifs}

Nous indiquons dans le tableau I les caractères métriques de ces populations et dans le tableau II, leurs caractères descriptifs.

D'après ces tableaux, on constate que, au Sahara, la stature des habitants, nomades ou sédentaires des oasis du Sahara septentrional, s'abaisse sensiblement par rapport aux habitants du nord de l'Afrique (Algériens). Elle est particulièrement basse chez les Mozabites de Ghardaïa et chez les Mekhadma de Ouargla. Elle s'élève un peu chez les Chaamba nomades et dans l'ancienne communauté juive de Ghardaïa, qui a disparu en 1962. Ces données montrent qu'il existe incontestablement dans le Nord-Sahara des îlots de basse stature dont il serait intéressant de rechercher la cause. Il pourrait s'agir d'un effet d'endogamie qui tend à abaisser la stature chez les communautés refermées sur elles-mêmes et ne se mélangeant pas. La stature redevient plus élevée chez les nomades du Sahara central et méridional, Zenata, Reguibat, et surtout les Touaregs occidentaux dont la haute taille contraste nettement avec celle des nomades nordsahariens.

La largeur des épaules varie sensiblement. Les Mozabites et les nomades du NordSahara ont les épaules les plus étroites, fait qui confirme la gracilité de leurs proportions corporelles. Les Reguibat de l'Ouest saharien ont, eux aussi, un buste peu développé. Les Zenata et les Touaregs se situent entre ces deux extrêmes. Les sédentaires Mrabtines de l'Ahaggar se rapprochent des sédentaires du Tell algérien.

111 Les dimensions relatives du bassin classent presque tous les groupes, sauf les nomades et sédentaires du Sahara central et méridional (Reguibat, Zenata, Mrabtines, Touaregs) dont le bassin est étroit, dans les valeurs moyennes de la classification de l'indice acromio-iliaque (troncs intermédiaires), mais avec des chiffres généralement plus élevés que ceux des Algériens dont la forme du tronc est plus trapézoïdale. Les formes rectangulaires prédominent chez les Reguibat, dans les oasis du Sahara septentrional, les Mrabtines et les Touaregs.

Tableau I. Caractères métriques des groupes ethniques du Sahara. 
A. Caractères corporels $(\mathrm{en} \mathrm{cm}$ )

\begin{tabular}{|l|r|c|c}
\hline & N & M & E.T. \\
\hline Stature : & 50 & 162 & \\
Mozabites (Amat) & 70 & 164 & - \\
Mozabites (Chantre) & 36 & 166 & 5,06 \\
Juifs (Ghardaïa) & 130 & 165,9 & 6,20 \\
Chaamba & 140 & 163 & 6,04 \\
Mekhadma & 199 & 166,8 & 6,05 \\
Reguibat & 31 & 168,7 & 5,75 \\
Zenata & 45 & 168,2 & 6,64 \\
Mrabtines & 90 & 174,2 & 6,81 \\
Touaregs ouest & 70 & 170,9 & - \\
Touaregs est & & & \\
Largeur des épaules : & 50 & 31,4 & - \\
Mozabites & 36 & 37 & 2,19 \\
Juifs (Ghardaïa) & 130 & 33,1 & 1,94 \\
Chaamba & 139 & 33,0 & 1,88 \\
Mekhadma & 199 & 32,9 & 1,91 \\
Reguibat & 31 & 36,7 & 1,65 \\
Zenata & 44 & 38,2 & 2,60 \\
Mrabtines & 71 & 37,1 & 2,40 \\
Touaregs ouest & 65 & 37,3 & - \\
Touaregs est & & & \\
\hline
\end{tabular}

\begin{tabular}{|l|r|r|c|}
\hline Largeur du bassin : & 50 & 28,1 & - \\
Mozabites & 36 & 28,8 & 1,90 \\
Juifs (Ghardaïa) & 130 & 28,4 & 1,73 \\
Chaamba & 139 & 27,6 & 1,40 \\
Mekhadma & 199 & 27,3 & 1,66 \\
Reguibat & 31 & 26,3 & 1,39 \\
Zenata & 44 & 26,6 & 1,37 \\
Mrabtines & 63 & 28,6 & 2,26 \\
Touaregs ouest & 65 & 26,3 & - \\
Touaregs est & & & \\
Périmètre du thorax : & 50 & 81,4 & - \\
Mozabites & 36 & 88,8 & 5,51 \\
Juifs (Ghardaïa) & 130 & 86,6 & 5,75 \\
Chaamba & 35 & 86,5 & 4,32 \\
Mekhadma & 199 & 87,9 & 4,71 \\
Reguibat & 43 & 87,5 & 5,20 \\
Mrabtines & & & \\
\hline
\end{tabular}




\section{B.- Caractères de la tête (en $\mathrm{cm}$ )}

\begin{tabular}{|c|c|c|c|}
\hline $\begin{array}{l}\text { Longueur de la tête : } \\
\text { Mozabites (A.) } \\
\text { Mozabites (C.) } \\
\text { Juifs (Ghardaïa) } \\
\text { Chaamba } \\
\text { Mekhadma } \\
\text { Reguibat } \\
\text { Zenata } \\
\text { Mrabtines } \\
\text { Touaregs ouest } \\
\text { Touaregs est } \\
\end{array}$ & $\begin{array}{r}50 \\
70 \\
36 \\
130 \\
125 \\
199 \\
31 \\
45 \\
93 \\
-\end{array}$ & $\begin{array}{c}190 \\
? \\
195,5 \\
196,5 \\
193,1 \\
197,5 \\
188,2 \\
194,6 \\
194,9 \\
- \\
\end{array}$ & $\begin{array}{l}: \\
5,71 \\
6,65 \\
6,47 \\
6,23 \\
5,90 \\
6,64 \\
7,24 \\
-\end{array}$ \\
\hline $\begin{array}{l}\text { Largeur de tête : } \\
\text { Mozabites (A.) } \\
\text { Mozabites (C.) } \\
\text { Juifs (Ghardaïa) } \\
\text { Chaamba } \\
\text { Mekhadma } \\
\text { Reguibat } \\
\text { Zenata } \\
\text { Mrabtines } \\
\text { Touaregs ouest }\end{array}$ & $\begin{array}{r}50 \\
70 \\
36 \\
130 \\
122 \\
199 \\
31 \\
45 \\
93 \\
\end{array}$ & $\begin{array}{c}147 \\
? \\
140,7 \\
142,9 \\
142,3 \\
145,8 \\
142,6 \\
146,8 \\
145,7 \\
\end{array}$ & $\begin{array}{l}- \\
4,28 \\
4,78 \\
5,00 \\
4,84 \\
5,52 \\
4,05 \\
4,66\end{array}$ \\
\hline $\begin{array}{c}\text { Indice céphalique : } \\
\text { Mozabites (A.) } \\
\text { Mozabites (C.) } \\
\text { Juifs (Ghardaïa) } \\
\text { Chaamba } \\
\text { Mekhadma } \\
\text { Reguibat } \\
\text { Zenata } \\
\text { Mrabtines } \\
\text { Touaregs ouest } \\
\text { Touaregs est }\end{array}$ & & $\begin{array}{l}77,3 \\
76,8 \\
72 \\
73,0 \\
74,0 \\
73,9 \\
75,8 \\
75,6 \\
74,9 \\
74,7\end{array}$ & $\begin{array}{l}- \\
3,20 \\
2,71 \\
2,93 \\
3,05 \\
3,25 \\
2,44 \\
3,25\end{array}$ \\
\hline $\begin{array}{l}\text { Hauteur de la face : } \\
\text { Mozabites } \\
\text { Juifs (Ghardaïa) } \\
\text { Chaamba } \\
\text { Mekhadma } \\
\text { Reguibat } \\
\text { Mrabtines } \\
\text { Touaregs ouest }\end{array}$ & $\begin{array}{r}50 \\
36 \\
130 \\
24 \\
193 \\
45 \\
63\end{array}$ & $\begin{array}{l}120,0 \\
123,4 \\
117,4 \\
119,0 \\
118,9 \\
122,5 \\
119,6\end{array}$ & $\begin{array}{c}- \\
5,97 \\
7,50 \\
5,61 \\
6,98 \\
6,90 \\
8,13\end{array}$ \\
\hline
\end{tabular}

\begin{tabular}{|c|c|c|c|}
\hline $\begin{array}{l}\text { Largeur face : } \\
\text { Mozabites } \\
\text { Juifs (Ghardaïa) } \\
\text { Chaamba } \\
\text { Mekhadma } \\
\text { Reguibat } \\
\text { Zenata } \\
\text { Mrabines } \\
\text { Touaregs ouest }\end{array}$ & $\begin{array}{r}50 \\
36 \\
130 \\
35 \\
159 \\
29 \\
45 \\
81\end{array}$ & $\begin{array}{l}133,0 \\
132,9 \\
132,7 \\
130,1 \\
133,5 \\
127,6 \\
134,9 \\
135,2\end{array}$ & $\begin{array}{l}5,57 \\
4,84 \\
4,39 \\
4,32 \\
5,16 \\
5,39 \\
4,96\end{array}$ \\
\hline $\begin{array}{l}\text { Largeur du front: } \\
\text { Mozabites } \\
\text { Juifs (Ghardaĩa) } \\
\text { Reguibat } \\
\text { Touaregs ouest }\end{array}$ & $\begin{array}{r}50 \\
36 \\
199 \\
45\end{array}$ & $\begin{array}{l}110,0 \\
105,8 \\
106,5 \\
108,3\end{array}$ & $\begin{array}{l}5,00 \\
4,35 \\
7,04\end{array}$ \\
\hline $\begin{array}{l}\text { Largeur des máchoires : } \\
\text { Juifs (Ghardaia) } \\
\text { Touaregs ouest }\end{array}$ & $\begin{array}{l}36 \\
29 \\
29\end{array}$ & $\begin{array}{l}105,4 \\
101,4\end{array}$ & $\begin{array}{l}7,09 \\
6,71\end{array}$ \\
\hline $\begin{array}{l}\text { Indice facial morph. } \\
\text { Mozabites } \\
\text { Juifs (Ghardaĩa) } \\
\text { Chaamba } \\
\text { Mekhadma } \\
\text { Reguibat } \\
\text { Mrabtines } \\
\text { Touaregs ouest } \\
\text { Touaregs est }\end{array}$ & $\begin{array}{r}\mathbf{N} \\
50 \\
36 \\
130 \\
125 \\
199 \\
45 \\
93\end{array}$ & $\begin{array}{l}M \\
90,2 \\
93,0 \\
87,9 \\
90,8 \\
89,1 \\
90,4 \\
88,3 \\
91,5\end{array}$ & $\begin{array}{l}\text { E.T. } \\
4,65 \\
5,12 \\
4,44 \\
4,14 \\
6,46\end{array}$ \\
\hline $\begin{array}{l}\text { Hauteur du nez: } \\
\text { Mozabites } \\
\text { Juifs (Ghardaĩa) } \\
\text { Chaamba } \\
\text { Mekhadada } \\
\text { Reguibat } \\
\text { Zenata } \\
\text { Mrabtines } \\
\text { Touaregs ouest }\end{array}$ & $\begin{array}{r}50 \\
36 \\
130 \\
35 \\
199 \\
31 \\
45 \\
89\end{array}$ & $\begin{array}{l}56,0 \\
55,8 \\
48,7 \\
48,9 \\
51,4 \\
47,0 \\
53,8 \\
53,7\end{array}$ & $\begin{array}{l}3,81 \\
4,45 \\
2,93 \\
3,25 \\
3,54 \\
4,45 \\
4,91\end{array}$ \\
\hline $\begin{array}{l}\text { Largeur du nez : } \\
\text { Mozabites } \\
\text { Juifs (Ghardäa) } \\
\text { Chaamba } \\
\text { Mekhadma } \\
\text { Reguibat } \\
\text { Zenata } \\
\text { Mrabtines } \\
\text { Touaregs ouest }\end{array}$ & $\begin{array}{r}50 \\
36 \\
130 \\
35 \\
159 \\
31 \\
45 \\
88 \\
\end{array}$ & $\begin{array}{l}34,0 \\
37,6 \\
35,8 \\
36,7 \\
34,3 \\
35,4 \\
39,9 \\
36,9\end{array}$ & $\begin{array}{l}2,87 \\
2,88 \\
2,91 \\
3,34 \\
2,72 \\
3,39 \\
3,23\end{array}$ \\
\hline $\begin{array}{l}\text { Indice nasal : } \\
\text { Mozabites } \\
\text { Juifs (Ghardaia) } \\
\text { Chaamba } \\
\text { Mekhadma } \\
\text { Reguibat } \\
\text { Zenata } \\
\text { Mrabtines } \\
\text { Touaregs ouest } \\
\text { Touaregs est }\end{array}$ & $\begin{array}{r}50 \\
36 \\
130 \\
35 \\
199 \\
31 \\
45 \\
88\end{array}$ & $\begin{array}{l}60,4 \\
68,1 \\
73,8 \\
74,5 \\
66,7 \\
75,1 \\
74 \\
68,6 \\
67,4\end{array}$ & $\begin{array}{l}5,63 \\
8,28 \\
5,36 \\
6,84 \\
7,11 \\
6,62 \\
8,54\end{array}$ \\
\hline
\end{tabular}

112 En ce qui concerne le thorax, l'aplatissement antéro-postérieur est plus marqué chez les habitants nomades du Sahara et les sédentaires d'Idèles que chez les Algériens du nord. Aussi, le périmètre relatif du thorax (périmètre/stature) est-il, chez eux, sensiblement plus faible, ainsi que chez les Mozabites sédentaires et les Juifs de Ghardaïa. Quant à la longueur du membre inférieur, il existe peu de données : seules 
trois séries, Chaamba, Mekhadma et Mrabtines, indiquent une tendance à une longueur relative assez élevée par rapport à la hauteur du buste, caractéristique des habitants des régions désertiques.

Pour l'indice céphalique (tableau I.B) les groupes les plus dolichocéphales sont les Juifs et les Chaamba de Ghardaïa et, d'une façon générale, les nomades sahariens, sauf les Zenata dont la tête est moins longue. Les Mozabites ont un indice mésocéphale indiquant une séparation ancienne et une endogamie par rapport à leurs voisins.

La largeur de la face est généralement étroite, en valeur absolue, chez les Sahariens, Mozabites inclus. La hauteur de la face est partout moins grande que chez les Algériens du nord dont la face est plutôt longue. Au Sahara, les faces assez longues et étroites prédominent mais la leptoprosopie y est moins accentuée que dans les régions côtières de l'Algérie. Les mâchoires sont larges chez les Juifs de Ghardaïa. Elles sont moins développées chez les Touaregs occidentaux.

Une grande hauteur du nez caractérise les habitants du nord de l'Afrique. On la retrouve chez les habitants sédentaires de Ghardaïa, et les groupes Touaregs et Mrabtines de l'Ahaggar. Les nomades sahariens, Chaamba, Mekhadma, Zenata, ont le nez moins haut. Les largeurs sont équivalentes. Du fait des variations de la hauteur, les indices nasaux différent selon les populations. Les nez sont relativement larges indice 70 à 75 - chez les Chaamba de Ghardaïa, les Mekhadma de Ouargla, les Zenata de Tindouf et les Mrabtines d'Idelès. Il est intéressant de noter que des types variés peuvent coexister dans une aire assez restreinte comme les oasis de Ghardaïa et de Ouargla. Les quatre séries provenant de ces régions ont des moyennes différents, allant des nez très étroits des Mozabites aux nez plus larges des Mekhadma et des Chaamba. De même, plus au sud, les Zenata et les Mrabtines diffèrent des Touaregs occidentaux et orientaux par un indice nasal plus élevé. Il semble que l'on puisse imputer ce fait à un métissage avec des éléments négroïdes. Dans l'ensemble, on note une légère augmentation de l'indice nasal du nord au sud chez les populations leucodermes d'Afrique du nord.

Tableau II. Caractères descriptifs (en \%)

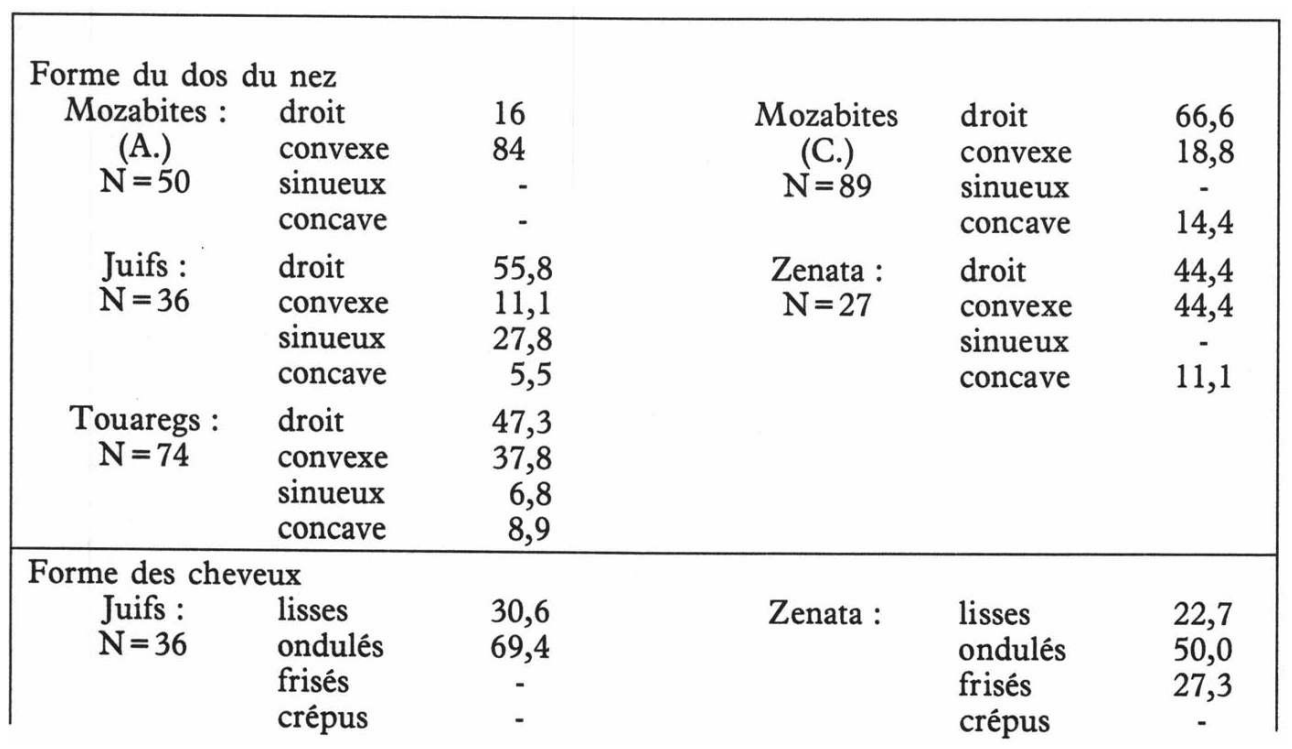




\begin{tabular}{|c|c|c|c|c|c|}
\hline $\begin{array}{c}\text { Touaregs O : } \\
\mathrm{N}=85\end{array}$ & $\begin{array}{l}\text { lisses } \\
\text { ondulés } \\
\text { frisés } \\
\text { crêpus }\end{array}$ & $\begin{array}{c}30,6 \\
53,0 \\
16,4 \\
-\end{array}$ & Touaregs E : & $\begin{array}{l}\text { lisses } \\
\text { ondulés } \\
\text { frisés } \\
\text { crépus }\end{array}$ & $\begin{array}{c}- \\
\text { généralement } \\
4,6 \\
-\end{array}$ \\
\hline \multicolumn{6}{|c|}{ Couleur des cheveux } \\
\hline $\begin{array}{l}\text { Mozabites : } \\
\mathrm{N}=50\end{array}$ & $\begin{array}{l}\text { blond foncé } \\
\text { châtains } \\
\text { brun-noir } \\
\text { noirs }\end{array}$ & $\begin{array}{c}- \\
32,0 \\
32,0 \\
36,0\end{array}$ & $\begin{array}{l}\text { Juifs : } \\
\mathrm{N}=36\end{array}$ & $\begin{array}{l}\text { blond foncé } \\
\text { châtains } \\
\text { brun-noir } \\
\text { noirs }\end{array}$ & $\begin{array}{l}- \\
11,2 \\
47,2 \\
41,6\end{array}$ \\
\hline $\begin{array}{l}\text { Zenata : } \\
\mathrm{N}=31\end{array}$ & $\begin{array}{l}\text { blond foncé } \\
\text { châtains } \\
\text { brun-noir }\end{array}$ & $\begin{array}{c}- \\
- \\
100,0\end{array}$ & $\begin{array}{c}\text { Touaregs } \mathrm{O} \\
\mathrm{N}=86\end{array}$ & $\begin{array}{l}\text { blond foncé } \\
\text { châtains } \\
\text { brun-noir }\end{array}$ & $\begin{array}{c}- \\
- \\
100,0\end{array}$ \\
\hline \multicolumn{6}{|c|}{ Couleur des yeux } \\
\hline $\begin{array}{l}\text { Mozabites: } \\
\qquad \begin{array}{l}\mathrm{N}=50\end{array}\end{array}$ & $\begin{array}{l}\text { bleus et } \\
\text { gris } \\
\text { marron } \\
\text { clair } \\
\text { marron } \\
\text { foncé }\end{array}$ & $\begin{array}{r}2,0 \\
30,0 \\
68,0\end{array}$ & $\begin{array}{l}\text { Juifs } \\
\mathrm{N}=36\end{array}$ & $\begin{array}{l}\text { bleus et } \\
\text { gris } \\
\text { mêlés vert/ } \\
\text { marron } \\
\text { marron } \\
\text { foncé }\end{array}$ & $\begin{array}{l}11,1 \\
16,7 \\
72,2\end{array}$ \\
\hline $\begin{array}{c}\text { Touaregs } \mathrm{O} \\
\mathrm{N}=57\end{array}$ & $\begin{array}{l}\text { bleus et } \\
\text { gris } \\
\text { marron } \\
\text { clair } \\
\text { marron } \\
\text { foncé }\end{array}$ & $\begin{array}{c}- \\
14,0 \\
85,9\end{array}$ & & & \\
\hline
\end{tabular}

Répartition des types de coloration d'après la formule de Livi : \% de yeux clairs (ou foncés) + \% de cheveux clairs (ou foncé) /2)

\begin{tabular}{|l|c|c|c|}
\hline & type clair & type mixte & type foncé \\
\hline Mozabites & 1,0 & 31,0 & 68,0 \\
Juifs & 5,5 & 13,9 & 80,5 \\
Touaregs ouest & - & 7,0 & 92,9 \\
\hline
\end{tabular}

Les profils rectilignes prédominent partout, mais les formes convexes sont cependant fréquentes chez les nomades sahariens. Chez les populations sédentaires de Ghardaïa, il semble que les nez convexes soient plus rares que chez leurs voisins du nord et du sud. Cepdendant, les fréquences très différentes trouvées par Amat et par Chantre chez les Mozabites montrent que des chiffres reposant sur une simple observation directe ne doivent être retenus qu'avec la plus grande réserve.

117 La forme des cheveux indique une prédominance des cheveux ondulés dans les trois séries à notre disposition. La couleur des cheveux montre une prédominance des cheveux brun noir et noirs chez les Juifs de Ghardaïa, les Zenata et les Touaregs de l'ouest. Une couleur un peu plus claire se rencontre chez les Mozabites (11\%). Il n'existe pas de cheveux roux parmi les populations sahariennes, comme on en rencontre (3,6\%) chez les Kabyles du nord.

118 La couleur des yeux varie davantage que la couleur des cheveux. Les teintes sombres prédominent chez les Mozabites et les Juifs de Ghardaïa, bien que les yeux marron clair soient bien représentés, et même des teintes claires chez les Juifs de Ghardaïa. Chez les Touraregs de l'Ouest, les teintes marron foncé sont prédominantes.

119 La combinaison de la couleur des cheveux et des yeux indique une prédominance des types foncés chez les trois séries sahariennes, avec, cependant une fréquence de 13,9\% de types mixtes chez les Juifs de Ghardaïa et de 5,5\% de types clairs. 


\section{Groupes sanguins}

La distribution des groupes $\mathrm{ABO}$ et Rh chez les Algériens du Tell et des Oasis du Sahara septentrional a été étudiée du point de vue régional dans un travail publié en 1971 (Benabadji et Chamla). Ce dernier peut être complété par d'autres études publiées entre 1934 et 1965. Le tableau III indique les répartitions phénotypiques et géniques du groupe $\mathrm{ABO}$, le tableau IV celles du système Rhésus.

L'hétérogénéité du système $\mathrm{ABO}$ chez les nomades et semi-nomades sahariens a été signalée à plusieurs reprises par les auteurs. Certains groupes Touaregs se distinguent par une fréquence très élevée du groupe 0 , indiquant une endogamie particulière que l'on peut rapprocher des fréquences trouvées chez les Amérindiens non métissés. Le gène $B$, de son côté, paraît diminuer du nord au sud de l'Algérie, de la zone tellienne vers les régions sahariennes. Les fréquences du groupe A sont relativement faibles, comparées à celles des Algériens du Tell.

En ce qui concerne le système Rhésus, les Algériens du nord sont caractérisés par une fréquence très élevée des sujets $\mathrm{Rh}$ positifs et une rareté des $\mathrm{Rh}$ négatifs. Chez les groupes sahariens du Nord et du Centre, Chaamba, Reguibat, Arabes de la Saoura et du Tidikelt, les proportions de sujets Rh négatifs sont comparables à celles des Algériens du Tell. En revanche, chez certains groupes Touaregs du sud, les taux de Rh négatifs apparaissent plus élevés (Ahaggar, Ajjer). Signalons néanmoins la discordance des résultats obtenus par Barnicot et Benabadji sur les Touaregs de l'Aïr avec des taux de Rh négatifs variant de 2 à $15 \%$. De telles différences entre les données publiées anciennement sur les Algériens du nord aussi bien que sur les Touaregs de l'Aïr et les données plus récentes doivent vraisemblablement être imputées à des erreurs de technique dans la détermination des Rh négatifs.

Tableau III. Fréquences phénotypiques et géniques du système $A B O$ (en \%)

\begin{tabular}{|c|c|c|c|c|c|c|c|c|c|}
\hline & $\mathrm{N}$ & A & B & $\mathrm{AB}$ & 0 & $\mathrm{p}$ & q & $\mathrm{r}$ & Auteurs \\
\hline Chaamba & 312 & 27,9 & 26,6 & , & 40,4 & 182 & 174 & .635 & Kossovitch, 1934 \\
\hline Chaa & & & & & & & .062 & .760 & \\
\hline Regui & 4 & 32,4 & 14,7 & 7 & 46,1 & .218 & .113 & .668 & Ruffié et al., 1962 \\
\hline Beni-C & 20 & 5 & 10,2 & 3,4 & 52,9 & .200 & .071 & .727 & er, 1933 \\
\hline $\begin{array}{l}\text { Figuig (Imaziren) } \\
\text { Saoura : }\end{array}$ & 212 & 24,5 & ,7 & 2,4 & 54,6 & .135 & .120 & .744 & Ruffié et al, 1966 \\
\hline & 293 & 22,9 & 20,1 & 2,4 & 54,6 & .135 & .120 & .744 & Ruffié et al., 1966 \\
\hline $\begin{array}{r}\text { Chle } \\
\text { Tidike }\end{array}$ & 71. & 31,0 & 7,0 & 1,4 & 60,5 & .177 & .043 & .778 & Ruffié et al., 1966 \\
\hline & 288 & 32,6 & 12,1 & 4,5 & 50,7 & .206 & .087 & .706 & Ruffié et al., 1963 \\
\hline & 57 & 8. & 14 & 1 , & 75, & .054 & .082 & .863 & \\
\hline Arat & 145 & 24 & 11 & 3 , & 60, & .15 & .078 & .768 & Ben \\
\hline $\begin{array}{l}\text { Touar } \\
\text { Ajjer : }\end{array}$ & 73 & 34,1 & 8,2 & 1,4 & 56,1 & .199 & .051 & .748 & ch, 1932 \\
\hline $\begin{array}{l}\text { Touaregs } \\
\text { Aïr : }\end{array}$ & 89 & 15,7 & 12,4 & 1,1 & 70,8 & .088 & .070 & .841 & andoul et Jacquemin, 1953 \\
\hline Tou & 164 & 23 & 11 & 1,8 & 62 , & 1 & .07 & .792 & $\mathrm{Ba}$ \\
\hline Touaregs & 95 & 22,1 & 10,5 & - & 67,3 & .118 & .054 & .828 & Benab \\
\hline
\end{tabular}


Tableau IV. Fréquences du système Rhésus (en \%)

\begin{tabular}{|c|c|c|c|c|c|c|}
\hline & $\mathrm{N}$ & $\mathrm{Rh}+$ & $\mathrm{Rh}-$ & $\mathrm{D}$ & $d$ & Auteurs \\
\hline Chaamba & 232 & 87,93 & 12,07 & .653 & .347 & Ruffié et al., 1962 \\
\hline Reguibat & 401 & 93,02 & 6,98 & .736 & .264 & Ruffié et al., 1960 \\
\hline \multicolumn{7}{|l|}{ Saoura : } \\
\hline Arabes & 293 & 90,10 & 9,90 & .685 & .315 & Ruffié et al., 1966 \\
\hline Chleuh & 71 & 90,14 & 9,86 & .686 & .314 & Ruffié et al., 1965 \\
\hline \multicolumn{7}{|l|}{ Ahaggar: } \\
\hline Touaregs & 57 & 85,96 & 14,04 & .626 & .374 & Benabadji et al., 1965 \\
\hline Arabes & 132 & 90,91 & 9,09 & .700 & .300 & Benabadji et al., 1965 \\
\hline \multicolumn{7}{|l|}{ Tidikelt : } \\
\hline Arabes & 268 & 94,78 & 5,22 & .772 & .228 & Ruffié et al., 1963 \\
\hline Ajjer : & & & & & & \\
\hline Touaregs & 89 & 70,79 & 29,21 & .460 & .540 & Jacquemin, 1952 in Mourant \\
\hline \multicolumn{7}{|r|}{10 , } \\
\hline Touaregs & 93 & 97,8 & 2,1 & .854 & .146 & Barnicot et al., 1954 \\
\hline Touaregs & 90 & 84,44 & 15,56 & .606 & .394 & Benabadji et al., 1965 \\
\hline
\end{tabular}

\section{Hémoglobines anormales, Thalassémies, Défaut en G-6-P.D.}

Un grand nombre d'hémoglobines anormales a été mis en évidence dans la zone tellienne de l'Algérie. Leur détection et leur distribution ont été étudiées principalement par Cabannes (1962) sur un nombre important de sujets. Sur un total de 17197 sujets musulmans d'origine connue, 306 , soit $1,78 \%$, ont été trouvés porteurs d'une hémoglobinopathie, compte non tenu des cas de thalassémie.

Au Sahara, différents groupes arabo-berbères ont été testés dans le Nord, dans la région de Tindouf, la Saoura, dans l'Ahaggar, le Tassili n'Ajjer, ainsi que chez les Toubous du Tibesti. Chez presque tous, sauf chez les Chleuhs de la Saoura et les Tadjacan de Tindouf, ont été trouvés des sujets, nombreux dans certains groupes, porteurs d'hémoglobinopathies diverses, notamment $\mathrm{Hb} \mathrm{S}$ (sicklémie), $\mathrm{Hb} \mathrm{C}, \mathrm{Hb}$ K. Les taux augmentent du nord au sud du Sahara : très faibles, chez les habitants de la région de Tindouf et chez les Mozabites (endogamie ?), le nombre des sujets porteurs d'Hb anormales est déjà plus élevé dans la région de la Saoura ( 2 à $10 \%$ ), chez les Touaregs et les Arabes de l'Ahaggar et les Ajjer (2,8 à 9,6\%), et surtout chez les Toubous du Tibesti $(12,9 \%)$.

Dans certaines populations, seule l'Hb S est présente, notamment au Tibesti et dans les Ajjer où $\mathrm{Hb} C$ paraît totalement absente. Celle-ci, par contre, est plus fréquente que l'Hb $\mathrm{S}$ dans le Sahara occidental. Selon Cabannes, l'Hb $\mathrm{C}$ aurait en effet une diffusion plus limitée en Afrique que le trait sicklémique (L'Hb C serait inexistante à l'est d'une ligne théorique allant du golfe du Gabès au delta du Niger). Le gène $\mathrm{Hb} \mathrm{K}$ semblerait plus rare parmi les populations sahariennes, où il a été décelé chez seulement deux sujets sur un total de 1620 , soit $0,012 \%$, qu'en Algérie du nord où sa fréquence atteint $0,14 \%$.

En ce qui concerne les thalassémies, rappelons que celles-ci, à la différence des hémoglobines anormales, qui sont dues à une modification de structure de la globine, sont conditionnées par des mutations portant, sans doute, sur les gènes régulateurs des proportions d'hémoglobines normales. La forme la plus courante ( $\beta$-thalassémie) se caractérise notamment en Europe méridionale et en Afrique du nord par une diminution globale de l'Hb A et l'augmentation de l'Hb A2, et plus faiblement de l'Hb F 
(A2 thalassémie). Les syndromes de la thalassémie sont très polymorphes et peuvent présenter des aspects divers. On distingue trois formes principales: la thalassémie majeure (maladie de Cooley) qui est la forme la plus complète et la plus grave dont les porteurs sont homozygotes et disparaissent généralement tôt ; une forme modérée, la thalassémie mineure transmise par un seul des géniteurs (forme hétérozygote) et qui entraîne des désordres cliniques et cytologiques; une forme latente, la thalassémie minime des sujets cliniquement sains, uniquement porteurs de la tare sanguine inapparente. La distribution de la thalassémie se superpose à peu près à celle des hémoglobinopathies. On retrouve des thalassémiques presque partout où l'on rencontre des porteurs de gènes d'Hb anormales avec, parfois, association avec $\mathrm{Hb} \mathrm{S}$ ou $\mathrm{Hb}$ C. En outre, il semble établi que la thalassémie est une maladie qui frappe les populations sous-alimentées.

Au Sahara, diverses enquêtes dans la Saoura, le Tassili n'Ajjer, l'Ahaggar et au M'Zab, ont révélé l'existence de foyers thalassémiques dans les deux premières régions. Dans les Ajjer, 24 sujets sur 330 examinés, soit 7,2\%, présentaient une $\mathrm{Hb}$ A2 augmentée, sans qu'on note de différence entre les deux groupes Touareg et Arabe. Dans la région de la Saoura, les thalassémiques sont moins fréquents, 1,4 à 3,4\% chez les Chleuhs et les Arabes, ou même, absents chez les Guenammia. Ils semblent également absents dans l'Ahaggar chez les Touaregs comme chez les Arabes (mieux alimentés ?), ainsi que chez les Mozabites.

LE DÉFAUT EN G-6-P.D. (glucose-6-phosphate déshydrogénase) qui consiste en un trouble métabolique de l'hématie, à l'origine d'anémies hémolytiques aiguës ou chroniques, est relativement fréquent chez les populations du Bassin méditerranéen où les taux peuvent s'élever jusqu'à $10 \%$ dans certaines régions comme l'Italie et même $34 \%$ dans les régions côtières de la Sardaigne. Cette anomalie enzymatique présente plusieurs variantes accompagnées ou non de manifestations cliniques. On ne possède de chiffres que pour l'Afrique septentrionale et non sur le Sahara. Dans la première, les taux de défaut en G-6-P.D. varient entre 0 et 4,8 \% (6\% sur 33 sujets des Oasis, selon Benabadji).

\section{Position anthropologique des Sahariens}

La comparaison des séries nomades entre elles par la formule $\Delta \mathrm{g}$ de Hiernaux donne des coefficients de divergence variables, indiquant une diversité manifeste. Les Touaregs occidentaux semblent se situer à l'écart de tous les autres groupes sédentaires et nomades (tableau IV). Les Reguibat se rapprochent des Chaamba et des Mekhadma, mais divergent considérablement des nomades Zenata de l'ouest saharien. Les Mekhadma sont proches des Chaamba. Les Zenata se tiennent à l'écart de tous les groupes (fig. 2).

130 Au total, il semble exister moins de divergence entre les groupes sédentaires, même éloignés géographiquement, que parmi les groupes nomades sahariens. Les coefficients de distance entre tous les groupes sédentaires varient de 67 à 254. Ceux des groupes nomades s'échelonnent entre 56 et 534 . On ne peut guère attribuer, a priori, à des processus adaptatifs cette disparité entre les groupes nomades, puisqu'ils vivent dans un milieu analogue et dans des conditions de vie probablement peu différentes. Peut-on l'expliquer par une origine différente, hypothèse qui paraît peu probable, mais difficile à vérifier? Plus vraisemblablement, mais les données anthropologiques sont alors insuffisantes pour répondre avec certitude; il s'agit là de phénomène de dérive 
génétique auxquels s'ajoutent peut-être des facteurs de consanguinité qui pourraient être à l'origine d'une diversification des caractères biologiques de ces groupes.

Un fait intéressant est l'éloignement marqué qui existe entre les Algériens du nord - et d'une façon générale les groupes sédentaires du nord de l'Afrique - et les habitants nomades du Sahara. Ces divergences montrent que le Sahara a joué le rôle d'une barrière anthropologique, isolant en outre les unes des autres les populations qui l'habitent, facteur favorable à l'apparition de caractères biologiques spécifiques.

Distance générale de Hiernaux entre différentes populations d'Afrique septentrionale
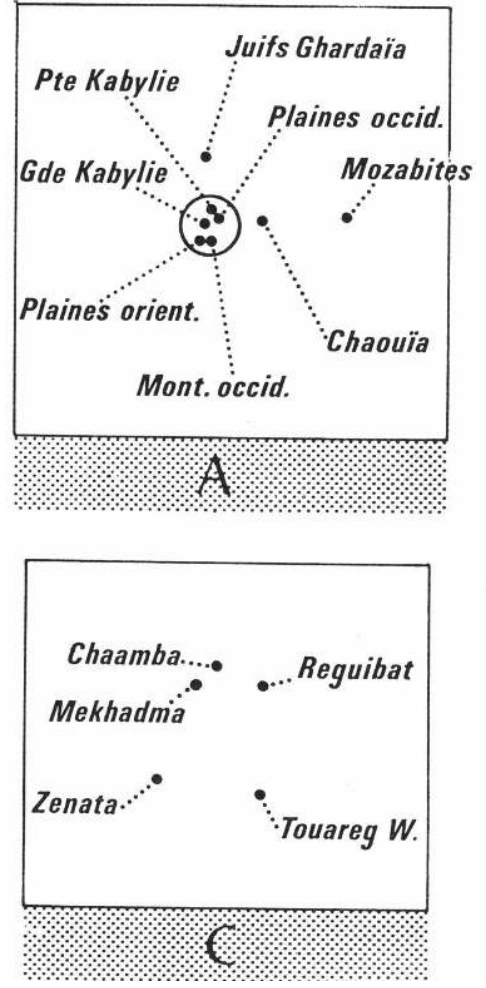

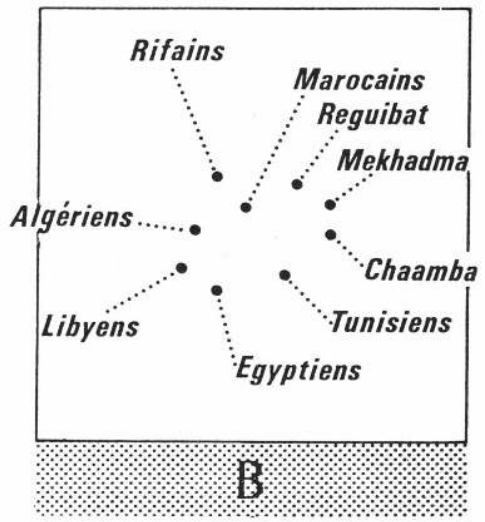

Distance générale de Hiernaux entre diffërentes populations d'Afrique septentrionale: A) entre Algériens sédentaires; B) entre les groupes sédentaires et nomades d'Afrique septentrionale; C) entre des groupes nomades du Sahara.

A) entre Algériens sédentaires ; B) entre les groupes sédentaires et nomades d'Afrique septentrionale ; c) entre des groupes nomades du Sahara.

\section{BIBLIOGRAPHIE}

Anthropologie physique des Marocains (D. Ferembach)

COON C.S., Tribes of the Rif Harvard, Afr. Studies, t. 9, Peabody Museum, Cambridge Mass.

GESSAIN M. « Les crêtes papillaires digitales de 194 Marocains », Bull. Mém. de la Soc. d'Anthrop. de

Paris, $\mathrm{X}^{\mathrm{e}}$ série, t. 8, 1957, p. 341-352.

KOSSOVITCH N., Anthropologie et groupes sanguins des populations du Maroc, Paris, Masson et Cie, 1953, $492 \mathrm{p}$. 
MOURANT A.E., The distribution of the human blood group, Oxford, Blackwell scientific publication, 1954, 438 p.

MOURANT A.E., KOPEC A.C., DOMANIEWSKA-SOBCZAK K., The A, B, O, blood groups. Comprehensive tables and maps of world distribution, Oxford, Blackwell scientific publications, 1958, $276 \mathrm{p}$.

\section{Anthropologie physique des Algériens (M.-C. Chamla)}

ALLISON A., Groupes sanguins ABO de 94 Kabyles de Tizi Ouzou, in Mourant, 1958.

AUZAS C, Groupes sanguins ABO de 15895 Musulmans d'Oran, in Mourant, 1958.

AUZAS C, « Les Flittas. Études ethnologique et sérologique », Bull. Soc. dAnthrop., Paris, 1957, t. 8, p. 329-340.

BARNICOT N., IKIN E., MOURANT A., « Les groupes sanguins ABO, MNS et Rh chez les Touareg de l'Air », L'Anthropologie, Paris, 1954, t. 58, p. 231-240.

BENABADJI M. et coll, « Étude hémotypologique des populations du massif du Hoggar et du plateau de l'Aïr. I. Les groupes érythrocytaires », Bull, et Mém. Soc. d'Anthrop., Paris, 1965, p. 171-180.

BENABADJI M., CHAMLA M.-C, « Les groupes sanguins ABO et Rh des Algériens », L'Anthropologie, 1971, $n^{\circ} 5-6$, p. 427-442.

BENHAMOU E., ZERMATI M., Groupes sanguins A, B, O de 487 Kabyles et Arabes, in Mourant, 1958.

BENOÎT F., KOSSOVITCH N., « Les groupes sanguins chez les Berbérophones (île de Djerba, Hoggar, Maroc) », C.R. Soc. de Biologie, 1932, t. 109, p. 198-200.

CAILLON L., DISDIER C, Les groupes sanguins d'une tribu berbère tunisienne, les Douiret, Arch. Inst. Pasteur, Tunis, 1930, t. 19, p. 50.

CHAMLA M.-C, « Les Algériens et les populations arabo-berbères du nord de l'Afrique », Mém. Rech. Anthrop., Archéo. et Ethnogr., Alger, n XXIV, 1974, 128 p.

CHAMLA M.-C, « Les empreintes digitales de 2336 Algériens musulmans », L'Anthropologie, 1961, $n^{\circ} 65$, p. 444-466.

CHAMLA M.-C, DEMOULIN F., « Les dermatoglyphes digito-palmaires des Chaouïas de Bou-zina » (Aurès, Algérie), Analyse intrapopulationnelle, L’Anthropologie, 1979, n 4, p. 626-664.

Coon C, Tribes of the Rif, Harvard African Studies, Cambridge, 1931, p. 417 p.

GHERIB B. et coll., « Études séro-anthropologiques XIII. Recherches sur l'île de Djerba, Tunisie », L'Anthropologie, Paris, 1965, t. 69, p. 511-518.

JOHNSON R., IKIN E. ET MOURANT A., Blood groups of the Aït Haddidu Berbers of Morocco, Human Biology, 1963, t. $35, \mathrm{n}^{\circ} 4$, p. 514-523.

KOSSOVITCH N., Anthropologie des groupes sanguins des populations du Maroc, Paris, Masson, 1953, $487 \mathrm{p}$.

LEVÊQUE J., « Les groupes sanguins des populations marocaines », Bull. Inst. d'Hygiène du Maroc, 1955 , t. 15 , p. 237.

MANDOUL R., JACQUEMIN P., Étude des groupes sanguins au Tassili n'Ajjer, Inst. Rech. Sahar., Univ. d'Alger, Mission scient, au Tassili n'Ajjer, I, 1953.

MÉCHALI D., In Mourant, 1976.

MESSERLIN A., Groupes sanguins et facteur Rhésus au Maroc, in Mourant, 1954. 
MOURANT A., The distribution of the human blood groups, Oxford Univ. Press, Londres, 1954, 438 p. MOURANT A. et coll., The ABO blood groups. Comprehensive tables and maps of world distribution, Blackwell publ. Oxford, 1958, 276 p.

MOURANT A. et coll., The distribution of the human blood groups and other polymorphisms, Oxford Univ. Press, Londres, 1976, 1055 p.

RANQUE J. et coll., Notions sommaires sur les groupes sanguins des populations tunisiennes, Transfusion, Paris, 1961, t. 4, p. 217-221.

RUFFIÉ J., CABANNES R., LARROUY G., « Étude hémotypologique des populations berbères de M'Sirda Fouaga (Nord-Ouest oranais) », Bull. Soc. d'Anthrop., Paris, 1962, t. 3, p. 294-314.

\section{Anthropologie physique des Tunisiens (M.-C. Chamla)}

BENOîT F., KOSSOVITCH N., « Les groupes sanguins chez les Berbérophones (îles de Djerba, Hoggar, Maroc) ", CR. Soc. de Biologie, 1932, t. 109, p. 198-200.

BERTHOLON L., CHANTRE E., Recherches anthropologiques dans la Berbérie orientale, Tripolitaine, Tunisie, Algérie, Lyon, 1913, 2 vol.

CHAMLA M.-C, SAHLY A., « Les empreintes digitales et palmaires des Tunisiens », Libyca, 1972, t. XX, p. 11-32.

COLLIGNON R., « Répartition de la couleur des yeux et des cheveux chez les Tunisiens sédentaires », Rev. d'Anthropologie, 1888, t. 3, p. 1-8.

EMBERGER J.-M., FOURMONT M., « Les dermatoglyphes digito-palmaires d'un groupe de Tunisiens », Journal de génétique humaine, 1970, t. 18, p. 1-19.

GHERIB B., Recherches séro-anthropologiques sur les populations tunisiennes, thèse de médecine, Marseille, 1962, et Bull. Soc. d'Anthrop., Paris, 1965, p. 165-170.

GHERIB B., «Études séro-anthropologiques XIII. Recherches sur l'île de Djerba, Tunisie ", L'Anthropologie, 1965, t. 69, p. 511-518.

MARIE A., MC AULIFFE L., «Étude anthropométrique de 136 indigènes tunisiens », C.R. Académie des Se, 1920 , t. 170 , p. 204-206.

MOULEC J., ABDELMOULA H., « Quelques données sur les groupes sanguins des Tunisiens », La semaine des Hôpitaux, 1954, t. 30, p. 3061-3062.

RANQUE J. et al, « Notions sommaires sur les groupes sanguins des populations tunisiennes », Transfusion, 1961, t. 4, p. 217-221.

Anthropologie physique des Libyens (M.-C. Chamla)

BERTHOLON L., CHANTRE E., Recherches anthropologiques dans la Berbérie orientale, Tripolitaine, Tunisie, Algérie, Lyon, 1913, 2 vol.

BIASUTTI R., « La posizione antropologica dei Berberi e gli elementi razziali della Libia », Annal. dell'Instit. Univ. Orient, di Napoli, 1949, t. 3, p. 127-141.

CIPRIANI L., " Abitanti e caratteri antropologici », in Il Sahara italiano parte prima, Fezzan e Oasi di Gat, Rome, 1937, p. 355-383.

FALCO C, « Sulle figure papillare dei polpastrelli delle dita nei Libici », Riv. di Antropologia, 1917-18, t. 22, p. 91-148. 
LEBLANC M., « Anthropologie et ethnologie. (Groupes sanguins de 592 Fezzanais) », Inst. Rech. Sahar. Univ. d'Alger, Mission scientifique au Fezzan, 1946, t. I.

MAZZELLA H., « Groupes sanguins ABO de 275 Abeidat de Cyrénaïque », in Mourant, 1958.

MOURANT A, KOPEC A, DOMANIEWSKA-SOBCZAK K., The ABO blood groups, Oxford, Blackwell scient, public, $1958,276 \mathrm{p}$.

PONS J., « Impresiones dermopapilares en varias poblaciones (Bantu de Natal, Berbères de Giado, Garaitas de Polonia y Dauada del Fezzan) », Trab. del Instit. Bernard de Sahagun, 1953, t. 14, n 1, $26 \mathrm{p}$.

PUCCIONI N., Antropometrica delle genti della Cirenaica, Florence, 1934, 2 vol. , 798 p.

RANDALL MC IVER D., WILKIN A., Libyan notes, Londres, 1901, 113 p.

Anthropologie physique des populations du Sahara (M.-C. Chamla)

AMAT C, Les Beni M’Zab. Anthropologie de l'Algérie », Revue d'Anthropologie, 1884, t. 7, p. 617-639.

ID., Le M'Zab et M'Zabites, Paris, 1888, 306 p.

BARNICOT N., Irin E., Mourant A., « Les groupes MNS et Rh chez les Touareg de l'Aïr »,

L'Anthropologie Paris, 1954, t. 58, p. 231-240.

BENABADJI M., « Le déficit en glucose-6-phosphate déshydrogénase dans le Bassin méditerranéen », Colloque intern. Soc. biol. humaine et de Trans, sanguine, 1968, Hammamet, Tunisie, 1970, p. 100-125.

BENABADJI M. et coll., « Étude hémotypologique des populations du massif du Hoggar et du plateau de l'Air », Bull. Soc. d'Anthrop., 1965,I, Les groupes érythrocytaires, p. 171-180. II. Les groupes sériques, p. 181-184.

BENABADJI M., CHAMLA M.-C, « Les groupes sanguins ABO et Rh des Algériens ", L'Anthropologie 1971, $\mathrm{n}^{\circ} 5-6$, p. 427-442.

BRIGGS C, «Contribution à l'anthropologie des Zenata du Sahara », Bull. Soc. d'Anthrop., Paris, 1955, t. 6, p. 214-222.

ID., « L'anthropologie des Touaregs du Sahara », Bull. Soc. d'Anthrop., Paris, 1955, t. 6, p. 93-116.

ID., " The living races of the Sahara desert », Pap. Peabody Mus. of Archeol. and Ethn., 1958, t. 28, n² , $217 \mathrm{p}$.

BRIGGS C, GUEDE N., « No more for ever, a Saharan Jewish town », Papers peab. Mus., 1964, t. 55, n 1, $108 \mathrm{p}$.

CABANNES R., « La hemoglobina C. Distribucion en el Norte y Oeste de Africa. Incidencias antropologicas », Sangre, 1960, t. 5, p. 51-66.

ID., Étude des types hémoglobiniques rencontrés dans les populations de la partie occidentale du continent africain (Maghreb, Sahara, Afrique noire occidentale). Thèse, Faculté des Sciences, Toulouse, 1962.

CABANNES R., LARROUY G., SENDRAIL A., « Étude hémotypologique des populations du massif du Hoggar et du plateau de l'Aïr. III. Les hémoglobines ». Bull, et Mém. Soc. d'Anthrop., Paris, 1969, n² 2, p. $143-146$.

CABANNES R., RUFFIÉ J., « Les types hémoglobiniques dans la population des Massifs montagneux du Sahara central (Hoggar et Tibesti) », L'Anthropologie 1961, t. 65, n 5-6, p. 467-483.

CABANNES R., et al., «Étude hémotypologique des populations sédentaires de la Saoura (Sahara occidental). II, Les hémoglobines ». Bull, et Mém. Soc. d’Anthrop., 1969, nº 2, p. 139-142. 
CHAMLA M.-C, « Les Algériens et les populations arabo-berbères du nord de l'Afrique. Étude anthropologique. ", Mém. Centre rech. anthrop. préhist. et ethno. ", Alger, n XXIV, 1974, 128 p. DUCOS J., VERNET P., VERGNES H., « Étude hémotypologique des populations du Tidikelt », Bull, et Mém. Soc. d'Anthrop., Paris, 1965, p. 185-187.

HORRENBERGER R., « Recherches sur les groupes sanguins dans le Sahara oranais », Arch. Institut Pasteur d'Algérie, 1933, t. 11, p. 433-444.

IGNAZI G., Documents anthropologiques sur la population d'Idelès, Hoggar. 69-XV inédit, C.N.R.S., R.C.P., $151,1970$.

JACQUEMIN, « Groupes sanguins ABO et Rh de 89 Touareg du Tassili n'Ajjer », in Mourant, 1954.

KOSSOVITCH N., « Recherches séro-anthropologiques chez quelques peuples du Sahara français », C.R. Soc Biologie, 1934, t. 116, p. 759-761.

LEBLANC M., « Anthropométrie et caractères morphologiques des Zenata sahariens », Revue anthropologique, 1934, t. 44, p. 338-349.

LEBLANC M.,-BERGEROT J., « Nouvelles contributions à l'étude de l'anthropologie anatomique des Touaregs », Revue Anthropologique, 1936, t. 46, p. 140-150.

LEFEVRE P., CABANNES R., SENDRAIL A., « Étude hémotypologique des populations du Tassili n'Ajjer », Bull, et Mém. Soc. d'Anthrop., Paris, 1967, n 4, p. 419-439.

LHOTE H., « Contribution à l'anthropologie somatique des Touaregs », Revue anthrop., 1938, t. 48, p. 284-306.

MANDOUL R., JACQUEMIN P., «Étude des groupes sanguins au Tassili n'Ajjer », Inst. rech. sahar. Univers. d'Alger, Mission scientifique au Tassili n'Ajjer, I, 1953.

MOURANT A., The distribution of the human blood groups, Oxford, 1954, 438 p.

RUFFIÉ J., BENABADJI M., LARROUY G., «Étude hémotypologique des populations sédentaires de la Saoura (Sahara occidental). I. Les groupes sanguins érythrocytaires. ", Bull, et Mém. Soc. d'Anthrop., Paris, 1966, t. 9, p. 45-53.

RUFFIÉ J., CABANNES R., LARROUY G., « Étude hémotypologique des populations berbères de M'Sirda Fouaga (N.-O. oranais) », Bull, et Mém. Soc. d'Anthrop., Paris, 1962, t. 3, p. 294-314.

RUFFIÉ J., DUCOS J., LARROUY G., « Étude hémotypologique des populations Chaamba de la région du M’Zab (département des Oasis) », Bull. Soc. d'Anthrop., Paris, 1962, p. 354-371.

RUFFIÉ J., DUCOS J., VERGNES H., « Étude hémotypologique des populations du Tidikelt (Sahara central) », Bull. Soc. d'Anthrop., Paris, 1963, t. 4, p. 531-544.

SUAUDEAU C, Le défaut en glucose-6-phosphate déshydrogénase : revue de la littérature et étude en Algérie centrale, thèse de médecine, Marseille, 1965, 309 p.

WYSS-DURANT E., « Recherches anthropologiques dans le Tibesti occidental », Arch. Suis. d'Anthrop. génér., 1947-48, t. 13, p. 125-155.

\section{INDEX}

Mots-clés : Préhistoire, Anthropologie, Sahara, Protohistoire, Maghreb, Origines, Histoire contemporaine 